\title{
Electron thermalization and relaxation in laser-heated nickel by few-femtosecond core-level transient absorption spectroscopy
}

\author{
Hung-Tzu Chang $\odot,{ }^{1, *}$ Alexander Guggenmos $\odot,{ }^{1, *}, \dagger$ Scott K. Cushing,,${ }^{1,2, \ddagger}$ Yang Cui,,${ }^{3,4, \S}$ Naseem Ud Din $\odot,{ }^{5}$ \\ Shree Ram Acharya, ${ }^{5}$ Ilana J. Porter ${ }^{10},{ }^{1,2}$ Ulf Kleineberg, ${ }^{3,4}$ Volodymyr Turkowski, ${ }^{5}$ Talat S. Rahman ${ }^{(0,}{ }^{5}$ \\ Daniel M. Neumark (i), ${ }^{1,2, \|}$ and Stephen R. Leone (i) $1,2,6, \mathbb{\|}$ \\ ${ }^{1}$ Department of Chemistry, University of California, Berkeley, California 94720, USA \\ ${ }^{2}$ Chemical Sciences Division, Lawrence Berkeley National Laboratory, Berkeley, California 94720, USA \\ ${ }^{3}$ Max-Planck Institut für Quantenoptik, Hans-Kopfermann-Str. 1, 85748 Garching, Germany \\ ${ }^{4}$ Fakultät für Physik, Ludwig-Maximilians-Universität München, Am Coulombwall 1, 85748 Garching, Germany \\ ${ }^{5}$ Department of Physics, University of Central Florida, Orlando, Florida 32816, USA \\ ${ }^{6}$ Department of Physics, University of California, Berkeley, California 94720, USA
}

(Received 28 September 2020; revised 7 December 2020; accepted 5 January 2021; published 10 February 2021)

\begin{abstract}
Direct measurements of photoexcited carrier dynamics in nickel are made using few-femtosecond extreme ultraviolet (XUV) transient absorption spectroscopy at the nickel $M_{2,3}$ edge. It is observed that the core-level absorption line shape of photoexcited nickel can be described by a Gaussian broadening $(\sigma)$ and a red shift $\left(\omega_{s}\right)$ of the ground-state absorption spectrum. Theory predicts and the experimental results verify that after initial rapid carrier thermalization, the electron temperature increase $(\Delta T)$ is linearly proportional to the Gaussian broadening factor $\sigma$, providing quantitative real-time tracking of the relaxation of the electron temperature. Measurements reveal an electron cooling time for $50 \mathrm{~nm}$ thick polycrystalline nickel films of $640 \pm 80 \mathrm{fs}$. With hot thermalized carriers, the spectral red shift exhibits a power-law relationship with the change in electron temperature of $\omega_{s} \propto \Delta T^{1.5}$. Rapid electron thermalization via carrier-carrier scattering accompanies and follows the nominal 4-fs photoexcitation pulse until the carriers reach a quasithermal equilibrium. Entwined with a $<6$ fs instrument response function, carrier thermalization times ranging from 34 fs to 13 fs are estimated from experimental data acquired at different pump fluences and it is observed that the electron thermalization time decreases with increasing pump fluence. The study provides an initial example of measuring electron temperature and thermalization in metals in real time with XUV light, and it lays a foundation for further investigation of photoinduced phase transitions and carrier transport in metals with core-level absorption spectroscopy.
\end{abstract}

DOI: 10.1103/PhysRevB.103.064305

\section{INTRODUCTION}

Probing and harnessing the relaxation of hot carriers in metals and semiconductors are vital to the development and design of photovoltaics and photocatalysts [1-7], and to the understanding of mechanisms in various photoinduced phase transitions [8-11]. After photoexcitation, carriers driven out of equilibrium quickly form a thermalized hot carrier distribution within a few to tens of femtoseconds through carrier-carrier scattering, before further cooling takes place through carrier-phonon interactions at timescales ranging from hundreds of femtoseconds to picoseconds [12]. Although the carrier cooling process typically involves complex

\footnotetext{
${ }^{*}$ These authors contributed equally to this work.

$\dagger$ Present address: UltraFast Innovations GmbH, Am Coulombwall 1, 85748 Garching, Germany.

${ }^{\ddagger}$ Present address: Division of Chemistry and Chemical Engineering, California Institute of Technology, Pasadena, CA 91125, USA.

${ }^{\S}$ Present address: Fakultät für Chemie, Technische Universität München, Lichtenbergstr. 4, 85748 Garching, Germany.

"dneumark@berkeley.edu

"Isrl@berkeley.edu
}

interactions between the electronic, phonon, and spin degrees of freedom, the dynamics can be successfully described phenomenologically by a "multitemperature model" in a wide variety of systems $[1,3,5,11-15]$. In such a model, the electronic, vibrational, and spin degrees of freedom are regarded as individual heat reservoirs and the energy transfer between the reservoirs is governed by a set of "interaction coefficients." Each reservoir is presumed to be in quasithermal equilibrium with a particular "temperature," based on the assumption that the heat equilibration within each reservoir, e.g., due to electron-electron scattering within electronic reservoir and anharmonic interactions for phonon baths, is much faster than the inter-reservoir energy transfer [15]. The usefulness of multitemperature models is widely evidenced in studies of energy transfer in heterostructures [16], hot electron cooling in two-dimensional materials and superconductors $[17,18]$, and photoinduced spin dynamics and phase transitions $[8,11,19$ 28].

Despite the success of the multitemperature model in elucidating a wide variety of photophysical phenomena, its applications are often limited to systems with an already thermalized carrier distribution. On the other hand, nonequilibrium hot carriers are known to facilitate charge separation 
dynamics in organic heterojunctions [4] and play an important role in plasmon-induced photocatalysis [6,7]. Studies of ultrafast demagnetization and all-optical magnetic state switching also demonstrate that the resulting spin dynamics can be coherently driven by nonthermal photoexcited carriers [10,2935]. The importance of measuring the electron thermalization process is further outlined in a recent optical pump-probe study on copper, which shows that the electron thermalization timescale is strongly dependent on the excitation fluence and at the low fluence limit, the thermalization timescale can become comparable to the electron-phonon scattering time [36]. To measure the photoexcited carrier distributions, timeresolved photoemission methods for valence electrons probe energy- and momentum-resolved carrier distributions in real time. However, the photoemission methods are restricted to timescales greater than tens of femtoseconds due to the relation between the energy bandwidth and the duration of the pulses that eject the photoelectrons, and thus have limited capacity in directly capturing carrier dynamics below 20 fs while maintaining $<0.2 \mathrm{eV}$ energy resolution, which is typically required to resolve carrier distributions in condensed matter. In addition, as alloy and multilayer structures are intrinsic to the construction of photovoltaics and many magnetic materials exhibiting photoinduced changes in magnetization [30-32,35,37,38], insight into the properties and performance of these materials can be obtained through understanding the carrier dynamics in each layer or subdomain in the system. Therefore, it is important to develop a unified experimental approach that provides element specificity and thus domain or layer selectivity, can interrogate the sub-10-fs dynamics of nonequilibrium carrier distributions, and is also capable of presenting key parameters such as carrier temperature after thermalization to facilitate the understanding of the interactions between the different degrees of freedom in photoexcited materials.

Core-level transient absorption (TA) spectroscopy in the extreme ultraviolet (XUV) has recently been developed and utilized to investigate carrier dynamics in semiconductors [39-49]. Exploiting the element specificity of this method, Cushing et al. investigated layer-specific carrier dynamics in a $\mathrm{Si}-\mathrm{TiO}_{2}-\mathrm{Ni}$ trilayer structure [49]. In studies on germanium [40], lead iodide [43], and lead halide perovskites [44], the energy distribution of the carriers and their relaxation can be directly extracted from XUV TA spectra. In addition, Volkov et al. utilized XUV TA spectroscopy to explore effects due to the change of electronic screening during photoexcitation of titanium [50]. However, despite numerous studies on electron dynamics in solids using core-level TA spectroscopy, the methodology to extract the energy distribution of photoexcited carriers or carrier temperature in metals from core-level TA spectra is still lacking. For many semiconductors with well-screened core holes, features of the core-level absorption spectra can be mapped onto the conduction band (CB) density of states (DOS) [40,43,44,46,51,52], and carrier dynamics can therefore be directly extracted from core-level TA measurements. In metals, by contrast, many-body interactions of electrons at the Fermi surface with the core hole potential strongly renormalize the spectral line shape of core-to-CB transitions [53], resulting in strong resonances at the absorption edge termed "edge singularities" [54-58]. As many-body interactions drastically reshape the core-level absorption spectra beyond the CB DOS, it is thus highly challenging in metals to unravel the carrier distributions and extract important parameters such as carrier temperatures using core-level absorption spectroscopy.

Here we employ nickel as a prototypical system and study the core-level TA spectra at the nickel $M_{2,3}$ edge around $67 \mathrm{eV}$ to develop a framework to understand the core-level TA spectra of metals, extract the electron temperatures, investigate the carrier cooling dynamics, and explore electron thermalization. Nickel is a ferromagnetic material exhibiting sub-picosecond demagnetization when irradiated with a femtosecond laser pulse and has been extensively studied $[8,19,21,30,35,38,59-$ 65]. Time-resolved photoemission measurements indicate that the photoexcited electrons in nickel thermalize on a sub-30-fs timescale [65] and electron-phonon relaxation times ranging from 200 fs to 1 ps have been derived from optical transient reflectivity and time-resolved second harmonic generation measurements [19,59-63]. On the other hand, ultrafast electron diffraction experiments show that a nonthermal phonon distribution persists over several picoseconds after optical excitation [66]. The many studies on photoexcited carrier dynamics in nickel thus provide suitable benchmarks for the methodology development here to reveal electron dynamics in metals using XUV core-level spectroscopy.

In this work, it is observed that the core-level absorption of laser-heated nickel can be described by a red-shifted and Gaussian broadened static absorption spectrum. In Sec. II A, the results of a set of power dependence measurements are shown and the resulting Gaussian broadening exhibits a linear dependence with respect to the electron temperature rise. In addition, the fitted spectral shift exhibits a power-law relationship with the electron temperature change. A theory for the linear relation between the Gaussian broadening and electron temperature change is derived in Sec. II B to complement the analyses, and Sec. III presents a conjecture based on analogy to the works on many-body interactions in the core-excited state after optical excitation [50] to explain the nonlinear relationship between the spectral shift and electron temperature. Section IIC displays the extraction of electron temperature from core-level TA spectra of photoexcited nickel according to the linear relationship between the spectral broadening and electron temperature rise, enabling real-time tracking of the carrier cooling process, and an electron cooling time of $640 \pm 80 \mathrm{fs}$ is obtained. The measurement results indicate that the contribution to spectral changes from phonon heating is negligible and the cause of the spectral red shift in all measurements within this work is purely electronic. In Sec. II D, fluence dependence measurements reveal a decrease of XUV TA signal rise time from 35 fs to $15 \mathrm{fs}$ as the final electron temperature, viz. the maximum electron temperature after thermalization, increases from $2100 \mathrm{~K}$ to $3100 \mathrm{~K}$. The measured fluence-dependent electron thermalization times are in good agreement with theoretical predictions [67]. By comparing the $<6$ fs long instrument response function with the $<40$ fs growth dynamics of the spectral features that become the core-level absorption profile of thermalized carrier distributions in the fluence dependence measurements, a range of electron thermalization times between $34 \mathrm{fs}$ and $13 \mathrm{fs}$ is obtained. 


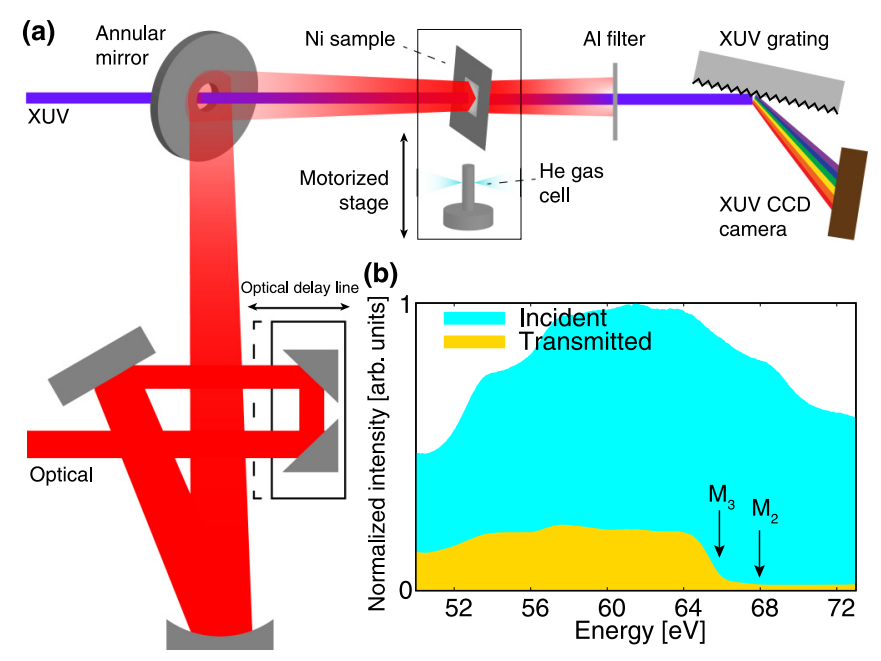

FIG. 1. (a) Experimental setup of the XUV TA experiment. (b) shows the spectra of incident and transmitted XUV pulses through the $50 \mathrm{~nm}$ thick Ni sample.

\section{RESULTS}

The core-level TA experiment on nickel was carried out with a table-top XUV source based on high-harmonic generation. The experiment is depicted in Fig. 1 and details of the experimental setup are described in Appendix A. Briefly, $50 \mathrm{~nm}$ thick polycrystalline nickel thin films deposited on $30 \mathrm{~nm}$ thick silicon nitride windows (Appendix B) were excited at normal incidence by a $4.3 \pm 0.2$ fs long (Gaussian FWHM), broadband optical pulse with a spectrum extending from $500 \mathrm{~nm}$ to $1000 \mathrm{~nm}$ and linear polarization. After optical excitation, the sample was probed by a time-delayed broadband linearly polarized XUV pulse, which is produced by high-harmonic generation in argon with a $3.6 \pm 0.1 \mathrm{fs}$ long laser pulse centered at $730 \mathrm{~nm}$. The polarization of the XUV pulse is parallel to the optical pump and the XUV spectrum spans 40-73 eV [Fig. 1(b)]. The XUV spectrum covers the nickel $M_{2,3}$ edges located at 66.2 and $68.0 \mathrm{eV}$, consisting of excitations from nickel $3 p$ levels to the conduction band [68].
The static absorption spectrum of nickel $M_{2,3}$ edges, shown in Fig. 2(a), exhibits a steep rising edge at approximately $66 \mathrm{eV}$ due to absorption from the Ni $3 p_{3 / 2}$ core level $\left(M_{3}\right)$ and another small absorption feature at approximately $68.5 \mathrm{eV}$ from the Ni $3 p_{1 / 2}$ level excitation $\left(M_{2}\right)$. Above $69 \mathrm{eV}$, the absorbance slowly decreases with increasing energy.

Dynamics following photoexcitation of nickel were probed by the change of core-level absorbance $\Delta A$ at variable time delays between the pump and probe pulses. A set of typical XUV TA spectra between -50 fs and 1.9 ps time delay is displayed in Fig. 2(b) alongside the static absorption spectrum in Fig. 2(a). Two positive features (increased absorption) are observed at $65.7 \mathrm{eV}$ and $67.4 \mathrm{eV}$, below the nickel $M_{3}$ and $M_{2}$ edges, respectively. The two features decay within $1 \mathrm{ps,} \mathrm{a}$ duration conforming to the electron cooling time in nickel due to electron-phonon interactions [63]. To probe the electron thermalization dynamics, the XUV TA results ranging from -20 fs to +35 fs time delay with 0.33 fs time steps are plotted in Fig. 2(c). The XUV TA spectra show no significant changes between 15 fs and 35 fs time delay and no energetically shifting spectral features are observed. In the following, we analyze the results by first considering the interpretation of the core-level TA spectra and extraction of electron temperature. Details of the electron cooling and thermalization dynamics are discussed in Secs. II C and II D, respectively.

To understand the core-level absorption spectra of optically excited nickel, we focus on the XUV TA profile right after photoexcitation. A core-level TA spectrum at 40 fs pumpprobe delay is plotted in Fig. 3(a). Here, apart from the increase in absorption (positive $\Delta A$ ) below the nickel $M_{3}$ and $M_{2}$ edges $(<67.4 \mathrm{eV})$, a shallow negative feature occurs above the nickel $M_{2}$ edge $(68 \mathrm{eV})$. Unlike the core-level TA spectra at the nickel $L_{2,3}$ edge, where the magnitude of absorption changes below and above the edge is highly symmetric [21,22], in the $M$ edge TA spectrum the positive features are much stronger than the negative feature and the integrated area of the TA profile $\left[\int \Delta A(\omega) d \omega\right]$ is clearly nonzero. The asymmetry of the TA profile indicates that it cannot be directly interpreted by electronic occupation below and above the Fermi level in contrast to the TA spectra at the nickel $L$ edge

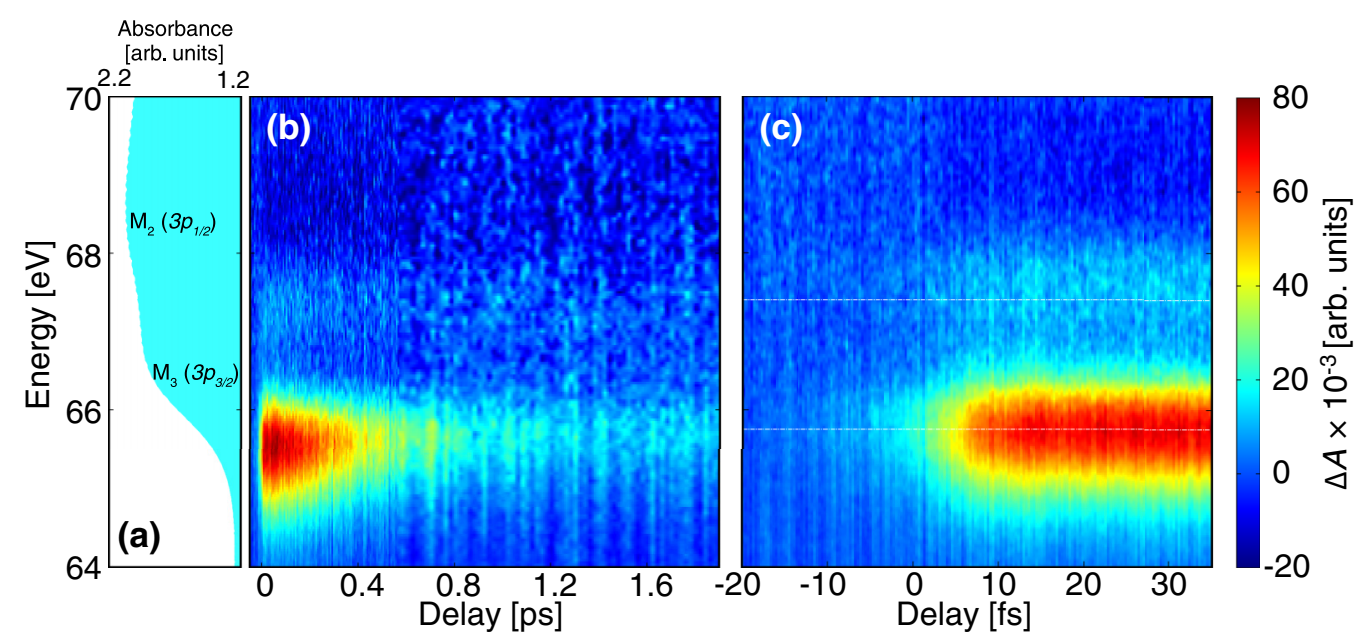

FIG. 2. (a) Static absorption spectrum of nickel $M_{2,3}$ edge and (b), the measured XUV TA spectra of nickel between -50 fs and 1.9 ps time delay. (c) displays the experimental XUV TA spectra between $-20 \mathrm{fs}$ and $35 \mathrm{fs}$ time delay. The experimental pump fluence of the results in (b) and (c) are 41 and $33 \mathrm{~mJ} / \mathrm{cm}^{2}$, respectively. 

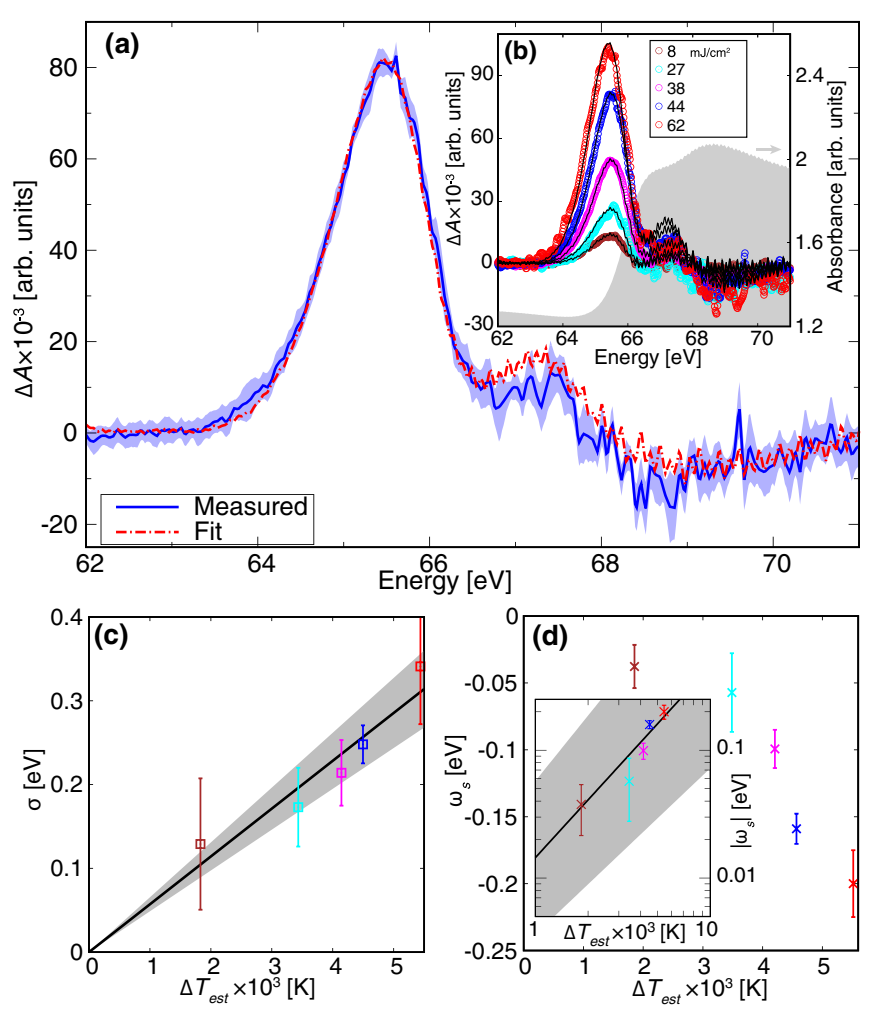

FIG. 3. (a) A typical XUV TA spectrum of nickel at $40 \mathrm{fs}$ time delay (blue line). $\triangle A$ denotes the change of XUV absorbance and the blue shade in (a) shows the uncertainty of the TA spectrum. The red line is the fitting result of Eq. (1) on the experimental data. The Gaussian broadening and shift obtained from the fitting are $0.25 \pm 0.02 \mathrm{eV}$ and $-0.16 \pm 0.01 \mathrm{eV}$, respectively. (b) Absorbance change $\Delta A$ (circles) after optical excitation at $40 \mathrm{fs}$ time delay with five different laser fluences. The static absorption spectrum of nickel $M_{2,3}$ edge is displayed in gray as a reference. Results of the fitting of measured data with Eq. (1) are shown in black lines and the obtained $\sigma$ and $\omega_{s}$ as a function of the simulated electron temperature rise $\Delta T_{\text {est }}$ are shown in (c) and (d), respectively. The results of linear fitting of $\sigma$ vs $\Delta T_{\text {est }}$ is displayed as a black line in (c) and the inset in (d) exhibits the fitting of $\left|\omega_{s}\right|$ vs $\Delta T_{\text {est }}$ with a power function (black line) in a log-log plot. The uncertainties in the fitting are shown as gray areas in (c) and (d), respectively.

[69]. While the cause of the asymmetric TA profile is beyond the scope of this work, it may stem from the asymmetric Fano broadening of the nickel $M_{2,3}$ edge due to Coster-Kronig decay of the core hole $[68,70,71]$, the many-body interactions between the electrons at the Fermi surface and the core hole [50], and the splitting of core levels with different angular and magnetic quantum numbers [72].

As Tengdin et al. showed that the electron thermalization time in nickel is $<30 \mathrm{fs},{ }^{1}$ a hot, thermalized electron distribution is expected to be established in the CB by $40 \mathrm{fs}$

\footnotetext{
${ }^{1}$ Tengdin et al. detected thermalized hot electron distribution with time-resolved angle-resolved photoemission 24 fs after photoexcitation by pulses centered at $780 \mathrm{~nm}$ with fluence of $<6 \mathrm{~mJ} / \mathrm{cm}^{2}$ [65]. The reported pulse duration in Ref. [65] is $28 \mathrm{fs}$. As the rate of electron scattering increases with carrier temperature, the carrier
}

after photoexcitation, and as the electron-phonon scattering time in nickel is on the order of a few hundred femtoseconds, energy loss to the phonon bath can be ignored. Almbladh and Minnhagen [73], Ohtaka and Tanabe [74-76], and Ortner and coworkers [77-79] have independently shown that the increase of electron temperature in metals can impose a broadening to the core-level absorption edge. In addition, the change of electronic screening in the $\mathrm{CB}$ due to photoexcitation can cause an energy shift of the core-to-CB transitions $[21,50,80]$. Thus motivated, we consider a model where the core-level absorption of photoexcited nickel $[I(\omega)]$ is simulated by a Gaussian broadening $\sigma$ of the static absorption spectrum $\left[I_{0}(\omega)\right]$ with an overall energy shift $\omega_{s}$ :

$$
\begin{aligned}
I\left(\omega, \omega_{s}, \sigma\right) & =\int d \omega^{\prime} I_{0}\left(\omega-\omega_{s}-\omega^{\prime}\right) f\left(\omega^{\prime}, \sigma\right), \\
f(\omega, \sigma) & =\frac{1}{\sigma \sqrt{2 \pi}} \exp \left(-\frac{\omega^{2}}{2 \sigma^{2}}\right) .
\end{aligned}
$$

The fitting of the experimental TA spectrum [Fig. 3(a), blue line] with Eq. (1) [Fig. 3(a), red line] exhibits good agreement between the fitted and the experimental result.

\section{A. Power dependence measurements}

To quantify the effects of electron temperature on the corelevel absorption spectra and extract the physical properties related to the broadening and shift, we performed power dependence measurements at five different laser fluences (8$62 \mathrm{~mJ} / \mathrm{cm}^{2}$ ). The core-level TA spectra at $40 \mathrm{fs}$ time delay and their fitting with Eq. (1) are displayed in Fig. 3(b). The magnitude of all TA features increases with increasing fluence and the fitting results show that Eq. (1) can accurately describe the measured spectral changes [Fig. 3(b), black lines]. The spectral broadening and shift obtained from the fitting with the corresponding electron temperature change, calculated from the pump fluence and electron heat capacity of nickel (Appendix C), are plotted in Figs. 3(c) and 3(d), respectively. Here it is observed that the spectral broadening $\sigma$ and the corresponding calculated electron temperature change $\Delta T_{\text {est }}$ can be fitted by a formula $\sigma=a \Delta T_{\text {est }}$ [Fig. 3(c), black line], with $a=(5.7 \pm 0.8) \times 10^{-5} \mathrm{eV} / \mathrm{K}$. The negative spectral shift $\omega_{s}$ indicates the absorption edge redshifts with increasing electron temperature [Fig. 3(d)] and it exhibits a power-law relationship with electron temperature change $\left(\omega_{s} \propto \Delta T_{\text {est }}^{\alpha}\right)$, with exponent $\alpha=1.5 \pm 0.2$ [Fig. 3(d), inset]. We defer discussion on the cause of the spectral shift to Sec. III A and first consider a theoretical explanation of the observed relation between the electron temperature change and Gaussian broadening.

\section{B. Relation between Gaussian broadening and electron temperature changes}

The many-body theory of core-level absorption in metals at nonzero temperatures, pioneered by Almbladh and Minnhagen [73], Ohtaka and Tanabe [74-76], and Ortner and

thermalization time is expected to be $<30 \mathrm{fs}$ long at fluences used in this study $\left(8-62 \mathrm{~mJ} / \mathrm{cm}^{2}\right)$. 
coworkers [77-79], shows that the core-level absorption spectra $I^{\prime}(\omega)$ can be approximated by a simple formula (in atomic units):

$$
\begin{aligned}
& I^{\prime}(\omega) \propto \frac{1}{2} \operatorname{Re} \int_{-\infty}^{\infty} e^{i\left(\omega-\omega_{0}\right) t} I^{\prime}(t) d t \\
& I^{\prime}(t)=\left(\frac{\pi T}{i \sinh (\pi T t)}\right)^{1-\zeta} .
\end{aligned}
$$

Here, $\omega_{0}$ represents the difference between the Fermi energy and the excited core level, $T$ is the electron temperature, and $\zeta<1$ is a coefficient related to the phase shift of the scattered electrons from the core hole potential. Although the expression only includes the effect of electron temperature, assumes a slowly varying density of states near the Fermi level, and ignores several factors that can lead to spectral distortion and broadening such as Auger decay of core holes, phonon heating [81,82], and sample crystallinity and inhomogeneity, it provides a clear mathematical representation of temperature effects on core-level absorption. Note that while the formula is derived from many-body theory, it is still valid for corelevel absorption in metals when core-hole mediated electron scattering at the Fermi surface is negligible.

To derive an expression relating spectral changes and electron temperature rise $\Delta T$, we consider an ansatz relating the absorption line shape $I\left(\omega, T_{0}+\Delta T\right)$ with electron temperature change $\Delta T$ to the absorption spectrum of the sample at temperature $T_{0}$ before excitation:

$$
I\left(\omega, T_{0}+\Delta T\right)=\int d \omega^{\prime} I\left(\omega-\omega^{\prime}, T_{0}\right) f\left(\omega^{\prime}, \Delta T\right),
$$

where the absorption line shape at electron temperature $T_{0}$ is convoluted by a function $f(\omega, \Delta T)$. The choice of convolution in the expression is motivated from experimental observation [Fig. 3 and Eq. (1)] and theoretical results [76]. The combination of Eqs. (2) and (3) indicate that $f(\omega, \Delta T)$ can be found by approximating the component of freeinduction decay $I^{\prime}(t)$ in Eq. (2) by

$$
\frac{\pi\left(T_{0}+\Delta T\right)}{\sinh \left(\pi\left(T_{0}+\Delta T\right) t\right)} \approx \frac{\pi T_{0}}{\sinh \left(\pi T_{0} t\right)} \times e^{g(t, \Delta T)},
$$

with $f(\omega, \Delta T)=\frac{1}{2 \pi} \int_{-\infty}^{\infty} e^{i \omega t} e^{(1-\zeta) g(t, \Delta T)} d t$. Using a secondorder expansion of $\Delta T$ on both sides of Eq. (4), it is shown that the first-order term in the expansion of $g(t, \Delta T)$ with respect to time $t$ is zero and the coefficient of the second order term is proportional to $(\Delta T)^{2}$ when $T_{0}$ is small compared to $\Delta T$ (Appendix D). In other words, the term $e^{(1-\zeta) g(t, \Delta T)}$ can be approximated by the expression

$$
e^{(1-\zeta) g(t, \Delta T)} \propto \exp \left(-\frac{(\sigma t)^{2}}{2}\right)
$$

with $\sigma=a \Delta T$, where $a$ is a proportionality constant. This suggests that the core-level absorption spectrum after laser heating $I\left(\omega, T_{0}+\Delta T\right)$ can be described by the convolution of the spectrum before heating $I\left(\omega, T_{0}\right)$ with a Gaussian function $f(\omega, \sigma)=\frac{1}{\sigma \sqrt{2 \pi}} \exp \left(-\frac{\omega^{2}}{2 \sigma^{2}}\right)$ and the broadening factor $\sigma$ is directly proportional to the temperature rise $\Delta T$.

Comparing Eq. (4) with Eq. (1), it is observed that the reference spectrum at temperature $T_{0}$ in Eq. (4) is represented by the static spectrum in Eq. (1). The two expressions merely differ by the spectral shift $\omega_{s}$, which is not present in the derivation above because the overall energy shift of the core-excited state is not included in either the many-body theory of core-level absorption [76,79] or our proposed ansatz [Eq. (3)]. The mathematical derivation thus justifies the fitting of TA profiles with a Gaussian broadened static spectrum and suggests that this approach can be extended to other metallic systems. In addition, the robustness of the method [Eq. (1)] is ensured by limiting the fitting parameters to only the shift and broadening. This is because the major factors that contribute to spectral distortions and broadenings are implicitly included in the formalism. The reference spectrum $I\left(\omega, T_{0}\right)$ in Eq. (3) and the static absorption spectrum in Eq. (1) automatically incorporate the spectral contribution from the intrinsic core hole lifetime as well as sample geometry and crystallinity, which remain unchanged throughout the measurement. The effects of interaction between the core hole and laser-heated CB electrons are included in Eq. (2), the starting point of the derivation of $f(\omega, \Delta T)$. Note, however, the mathematical derivation only considers the spectral changes due to variation in electron temperature; other contributions from processes that would follow photoexcitation, such as phonon dynamics, are not included. In addition, the derivation of $f(\omega, \Delta T)$ involves truncation of a series expansion and is not analytically exact. Therefore the application of this method [Eq. (1)] and the extraction of electron temperature from the broadening always require verification that the relation between the electron temperature and the broadening is linear and dynamics in the electronic domain are the dominant contributor to the TA line shape. The relationship between projected electron temperature and the fitted spectral broadening in power dependence measurements, and the correspondence between the fitted spectral shift and broadening, can both serve as checkpoints to examine the adequacy of this analysis approach.

\section{Electron cooling dynamics}

Equipped with the formalism to understand the spectral change in core-level excitations in nickel with hot thermalized electrons at a short 40 fs pump-probe delay [Eq. (1)], we consider here the electron cooling dynamics at $>40 \mathrm{fs}$ timescales [Fig. 2(b)]. To analyze the measured dynamics, we first apply Eq. (1) to fit the TA spectrum at each time delay. The fitted spectra are shown in Fig. 4(a), displaying good agreement with the experimental results [Fig. 2(b)]. Note that phonon heating is expected to occur from electron-phonon scattering processes during the cooling of the electron bath [63] and the adequacy of relating electron temperature with spectral broadening requires verification. It has been shown that the rise of nonthermal phonon occupation persists over timescales above 2 ps [66] and the electronic reservoir cools down below 1 ps [63]. If phonon dynamics contribute significantly to the XUV TA spectra, it is expected that at $>1$ ps timescales the contribution from phonons would dominate and the magnitude of the XUV TA signal would rise with increasing time delay. Contrary to expectations, however, the experimental TA signal diminishes to zero with increasing time delay and its magnitude at 1.9 ps delay is barely above noise level [Fig. 2(b)]. This suggests that the contribution from heated phonons to the observed core-level TA signal is negligible compared to 


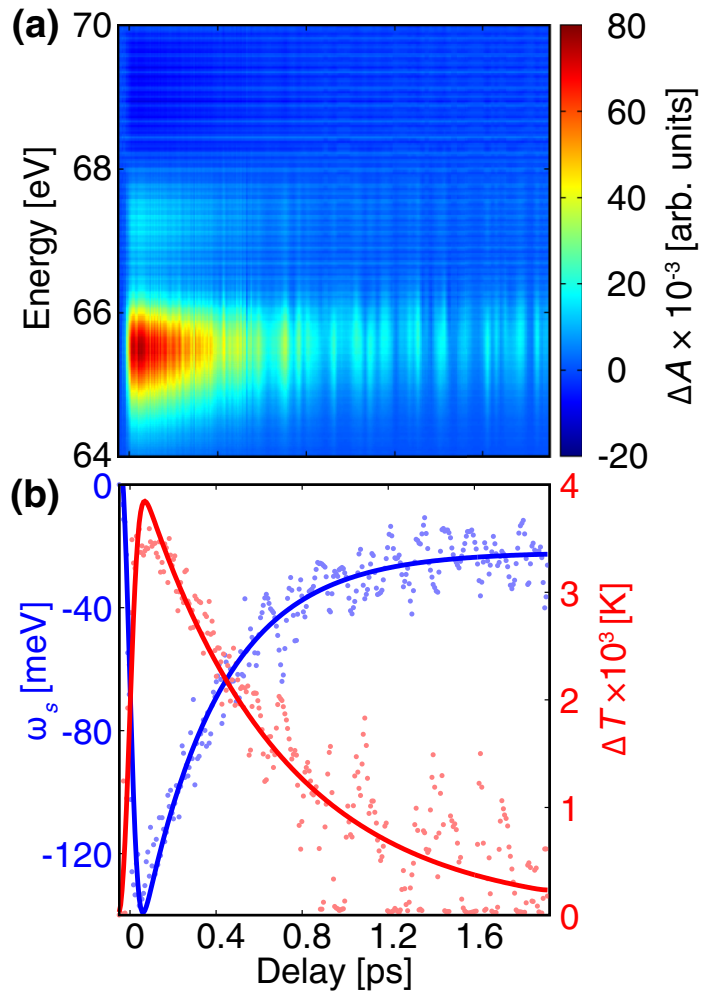

FIG. 4. (a) Results of fitting the TA spectra in Fig. 2(b) with Eq. (1) and the fitting parameters $\omega_{s}$ and $\Delta T$ at different time delays are shown as dots in (b). The fitting of the changes of $\omega_{s}$ and $\Delta T$ as a function of time delay with single exponential decay convoluted with a Gaussian instrument response function are shown as the blue and red line, respectively.

the dynamics in the electronic domain. ${ }^{2}$ As such, the obtained spectral broadening $\sigma$ can still be connected with $\Delta T$ in the analysis of XUV TA spectra at hundreds of femtoseconds to picoseconds.

Figure 4(b) shows the fitted spectral shift (blue dots) and electron temperature ( $\Delta T=\sigma / a$, red dots) derived from the fitted spectral broadening at different time delays. Both the spectral shift and electron temperature as a function of time delay can be fitted by a single exponential decay convoluted with the instrument response function [Fig. 4(b), lines] and the time constants for electron temperature decay $\tau_{\sigma}=640 \pm 80$ fs and spectral shift $\tau_{s}=380 \pm 30$ fs are obtained, respectively. Note that the shift $\omega_{s}$ and broadening $\sigma$ are the only variables in the fitting procedure [Eq. (1)], and no additional parameters are introduced. The $640 \pm 80 \mathrm{fs}$ cooling time is consistent with the reported values from optical pump-probe measurements $[59,60,62]$. The discrepancy between $\tau_{\sigma}$ and $\tau_{s}$ also agrees with the observed relationship between the electron temperature and the spectral shift in the power dependence measurements [Fig. 3(d)]. As the spectral shift is

\footnotetext{
${ }^{2}$ Note that the results only indicate that the TA signal is not sensitive to the particular phonon heating dynamics in the experiment. It does not imply that core-level absorption spectroscopy is insensitive to phonon dynamics overall and depending on the system measured, lattice dynamics can contribute to core-level TA signals (e.g., Ref. [85]).
}

related to the electron temperature change by a power law $\left(\omega_{s} \propto \Delta T^{\alpha}\right)$, it is expected that the decay dynamics of spectral shift follow the relation $\omega_{s}(t) \propto \Delta T(t)^{\alpha} \propto e^{-\alpha t / \tau_{\sigma}}$, with $\alpha=$ $\tau_{\sigma} / \tau_{s}$. Here, the obtained $\tau_{\sigma} / \tau_{s}$ is approximately 1.7 , agreeing with $\alpha=1.5 \pm 0.2$ obtained from the power dependence measurements [Fig. 3(d), inset]. It is thus observed that the relation between the spectral shift and broadening is maintained between $40 \mathrm{fs}$ and 1.9 ps time delay. At 40 fs delay, the effect of electron-phonon interaction is negligible because the timescale is well below the electron-phonon scattering time [63], whereas at picosecond timescales, nonequilibrium phonon dynamics are paramount $[63,66,83]$. The consistency of the behavior between the spectral shift and broadening from 40 fs to picosecond time delays further corroborate that the fitted spectral broadening and shift are related to physical properties in the electronic domain because the relation between the two persists regardless of the generation of phonons out of thermal equilibrium.

\section{Electron thermalization dynamics}

In this section, we focus on the few-femtosecond dynamics of carrier photoexcitation and thermalization. The XUV TA spectra between -20 fs and 35 fs pump-probe delay with optical pump fluence at $33 \mathrm{~mJ} / \mathrm{cm}^{2}$ are plotted in Fig. 2(c), which exhibits two positive features at $65.75 \mathrm{eV}$ and $67.4 \mathrm{eV}$ that increase in magnitude within -10 fs to +15 fs time delay and reach a plateau at $>15$ fs. The lack of changes in the TA signal between 15 fs and 35 fs suggests that the electrons thermalize within $15 \mathrm{fs}^{3}$ The absence of energetically shifting spectral features within -10 fs to +15 fs time delay, which could be used to directly signify the scattering and decay of the initial nonequilibrium photoexcited electrons to form a hot thermalized distribution, initially implies that the electrons thermalize within the pulse duration of the pump. However, analysis of the lineouts of TA features at $65.75 \mathrm{eV}$ and $67.4 \mathrm{eV}$ [Fig. 5(c) (dots)] shows that the duration of the growth of the TA features is significantly longer than the optical pump pulse. By fitting the lineouts with a modified Gaussian error function [Eq. (E1)], TA signal rise times $\tau_{\text {rise }}=15.1 \pm 0.4 \mathrm{fs}$ and $14 \pm 2$ fs for energies $65.75 \mathrm{eV}$ and $67.4 \mathrm{eV}$ are obtained, respectively [Fig. 5(c) (dashed lines)]. The cross-correlation time between the optical and XUV pulses, whose upper limit is set by the cross-correlation between the pump pulse and the driving field for high-harmonic generation, is less than $\sqrt{4.3^{2}+3.6^{2}} \approx 5.6 \mathrm{fs}$. The $15 \mathrm{fs}$ rise time of the features at $65.75 \mathrm{eV}$ and $67.4 \mathrm{eV}$ [Fig. 5(c)], which is much longer than the $<5.6$ fs cross-correlation time between the pump and the probe, suggests that the electron thermalization time is longer than the pump pulse duration and the lengthened rise time is connected to nonthermal electron relaxation. We present experimental evidence to support this hypothesis, and a conjecture on the cause of the absence of spectral signatures of nonequilibrium electron distribution is discussed in Sec. III B.

\footnotetext{
${ }^{3}$ Note that, however, because the core-level absorption spectrum at the Ni $M_{2,3}$ edge cannot be directly mapped on to the CB DOS, it is impossible to directly quantify the deviation of the carrier distribution from a hot Fermi-Dirac function with the core-level TA spectra.
} 

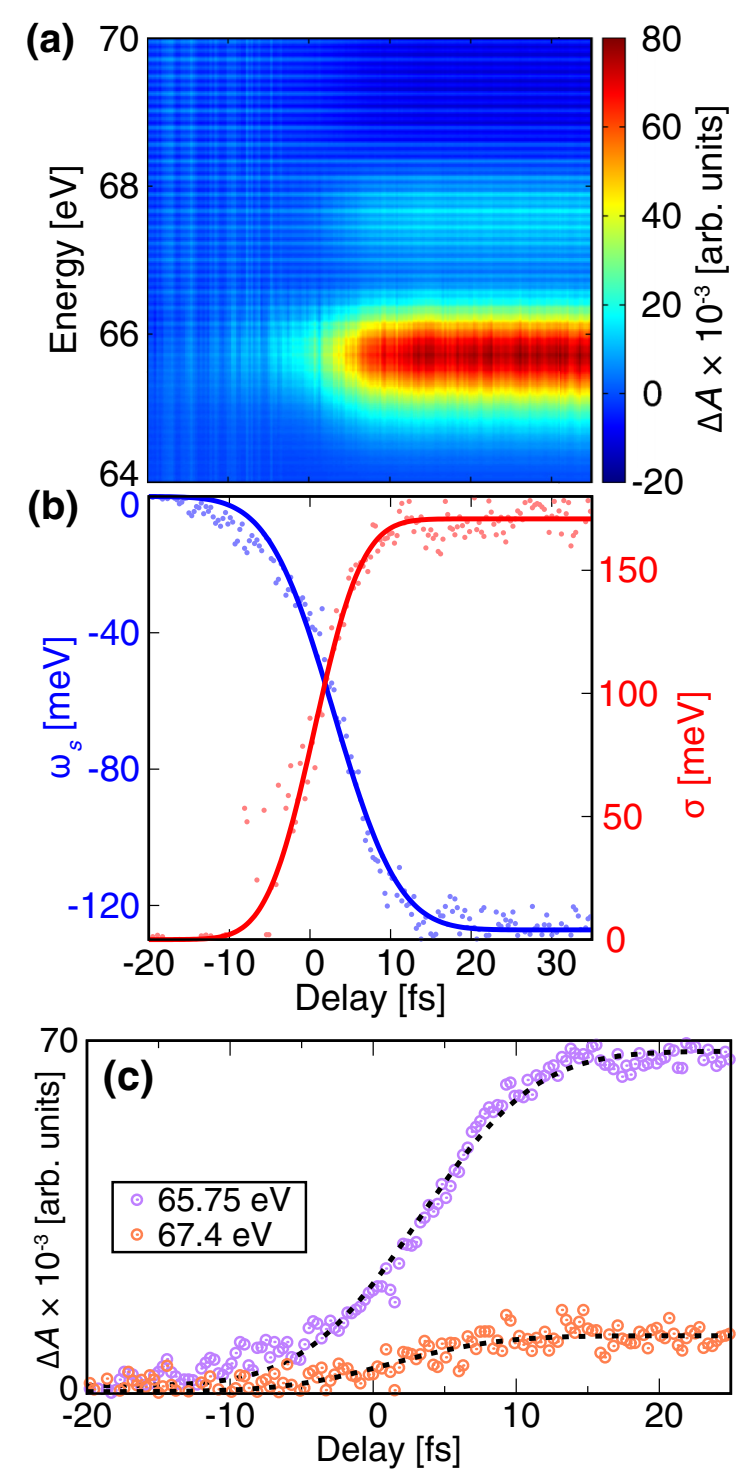

FIG. 5. (a) Fitting results of Fig. 2(c) with Eq. (1). The fitted edge shift $\left(\omega_{s}\right)$ and broadening $(\sigma)$ as a function of time delay are plotted in (b) as dots and the fitting of $\omega_{s}(t)$ and $\sigma(t)$ with Eq. (E1) are depicted as blue and red lines, respectively. (c) shows the lineouts of $\Delta A$, shown in circled dots, at $65.75 \mathrm{eV}$ and $67.4 \mathrm{eV}$ [Fig. 2(c), white dashed lines] and their fitting results (dashed black lines) with Eq. (E1).

To verify whether the electrons directly thermalize during photoexcitation, the XUV TA spectra [Fig. 2(c)] are fitted with Eq. (1), and the quality of the fitting and the relation between the fitted broadening $\sigma(t)$ and energy shift $\omega_{s}(t)$ are examined. Figure 5(a) shows the fitting results, and the fitted broadening and energy shift as a function of time delay are plotted in Fig. 5(b). The good agreement between the fitting results [Fig. 5(a)] and experimental data [Fig. 2(c)] initially suggests that the electrons thermalize within the duration of the optical pulse. However, if the electrons already thermalize within the timescale of photoexcitation, the resulting spectral broadening $\sigma$ and shift $\omega_{s}$ should follow the relation $\omega_{s}(t) \propto$ $\sigma(t)^{1.5}$ as shown in Sec. IIC. In Fig. 5(b), the changes in
TABLE I. Experimentally obtained TA signal rise time $\tau_{\text {rise }}$ and extracted electron thermalization time $\tau_{t h}$ vs electron temperature $T_{e}$ from fluence dependence measurements. The asymmetry in the uncertainty of $\tau_{\text {rise }}$ for entries with $T_{e}=2129 \mathrm{~K}$ and $2552 \mathrm{~K}$ is due to the drift of time delay within the experiment which cannot be compensated, leading to a stretch in the rise time. Further explanation on the time delay drift is described in Appendix A.

\begin{tabular}{lcc}
\hline \hline$T_{e}(\mathrm{~K})$ & $\tau_{\text {rise }}(\mathrm{fs})$ & $\tau_{\text {th }}(\mathrm{fs})$ \\
\hline 2129 & $35_{-15}^{+10}$ & 34 \\
2552 & $22_{-10}^{+5}$ & 21 \\
3060 & $15 \pm 3$ & 13 \\
\hline \hline
\end{tabular}

the broadening and the shift as a function of time delay are fitted to a modified Gaussian error function [Eq. (E1)] and the obtained error function rise times for the broadening and the shift are $\tilde{\tau}_{\sigma}=11.0 \pm 0.4$ fs and $\tilde{\tau}_{s}=15.0 \pm 0.3 \mathrm{fs}$, respectively. Given $\omega_{s} \propto \sigma^{1.5}$ for a thermalized electron distribution, the increase of magnitude in the spectral shift $\omega_{s}(t)$ should be steeper than the broadening $\sigma(t)\left(\tilde{\tau}_{\sigma}>\tilde{\tau}_{s}\right)$, which is opposite to the fitting results. This indicates that the electron thermalization time in nickel is either comparable or longer than the optical pulse ( $4.3 \mathrm{fs}$ ), and during the increase of magnitudes in the broadening and spectral red shift, the broadening $\sigma$ cannot be directly related to an electron temperature.

In addition to the inconsistent behavior of the spectral shift and broadening compared to the results obtained at $\geqslant 40 \mathrm{fs}$ time delay, we consider the fluence dependence of the TA signal rise time [Fig. 5(c)]. Fermi liquid theory indicates that the electron collision rate is proportional to the square of electron temperature [84]. Using Boltzmann collision integrals, Mueller and Rethfeld predict that the electron thermalization time in nickel decreases by an order of magnitude as the "final" electron temperature, viz. the electron temperature after thermalization, increases from $2000 \mathrm{~K}$ to $8000 \mathrm{~K}$ [67]. The fluence dependence of electron thermalization has been experimentally observed by Obergfell and Demsar in copper by optical pump-probe measurements [36]. Here we examine the connection between electron thermalization time and the rise time of the core-level TA signal [Fig. 5(c)] by a set of power dependence measurements at three different pump fluences. In Table I, we list the final electron temperatures $T_{e}$ and the rise times $\tau_{\text {rise }}$ of TA signal at $65.75 \mathrm{eV}$ and a clear decrease in the rise time with respect to increasing electron temperature is observed. As the final electron temperature rises from approximately $2100 \mathrm{~K}$ to $3100 \mathrm{~K}$, the measured rise time decreases from 35 fs to $15 \mathrm{fs}$. The observed behavior of the rise time as a function of final electron temperature is consistent with theoretical predictions of the electron thermalization time and we estimate this time $\tau_{t h}$ by deconvolving the growth dynamics with the pump-probe cross-correlation $\left(\tau_{t h} \approx \sqrt{\tau_{\text {rise }}^{2}-5.6^{2}}\right)$. The electron thermalization times obtained by deconvolution are listed in Table I, showing that the electron thermalization time decreases from 34 fs to 13 fs as the electron temperature rises from $2100 \mathrm{~K}$ to $3100 \mathrm{~K}$. The obtained thermalization times are on the same order of the theoretically predicted thermalization time in nickel [67]. 


\section{DISCUSSION}

Despite the success of fitting the TA profile with spectral shift and broadening [Eq. (1)], the physical origin of the spectral shift has yet to be clarified. In addition, while the electron thermalization time is extracted from the rise of TA signal [Fig. 5(c)], the absence of spectral signatures of the nonequilibrium electron distribution during photoexcitation has not been explained. Here we discuss the potential origin of the spectral shift and the absence of spectral features of nonthermalized electrons.

\section{A. Origin of spectral shift}

The spectral shift in the core-level absorption spectra can be interpreted as an overall change in the energy of the coreexcited state in laser-heated nickel. This can be caused by electron-phonon interactions [40], and in particular, lattice displacement and heating due to optical excitations [52,85]. However, as the experimental results suggest that the direct influence of phonon excitations on the TA profile is negligible, we restrict the discussion within the electronic domain. Electronically, the spectral red shift of core-level absorption after optical pump illumination can originate from the lowering of the chemical potential in the $\mathrm{CB}$ or stabilization of core-excited state due to many-body interactions [50]. The lowering of the chemical potential as a possible cause can be eliminated as Lin et al. and Colombier and coworkers showed that the chemical potential of the $\mathrm{CB}$ of nickel increases rather than decreases with rising electron temperature [86-88], in contrast to the experimental observations here. Due to the complexity in simulating the effect of many-body interactions in the core-excited state of nickel with hot, thermalized CB electrons, here we provide a subjective explanation for the nonlinear relationship between $\omega_{s}$ and $\Delta T$ based on related work by analogy and invite future theoretical work to verify the validity of the conjecture.

In attosecond TA studies of titanium, Volkov et al. showed that the optical excitation of electrons increases the occupation of the localized Ti $3 d$ orbitals, which further causes a spectral blue shift due to the increase of electronic repulsion in the core-excited state [50]. In nickel, the increase of electron temperature causes the transfer of $\mathrm{Ni} 3 d$ electrons to the higher-lying $4 s$ and $4 p$ bands [88], which would reduce electronic repulsion in the localized $3 d$ orbitals in contrast to the repulsion increase observed when heating an early transition metal such as Ti. The cause of the opposing behavior between $\mathrm{Ni}$ and $\mathrm{Ti}$ is theorized from the inversion in the orbital character with respect to band energies. In titanium, the occupied $4 s$ bands are below the largely unoccupied $3 d$ bands [50], whereas in nickel, the occupied bands are composed primarily of $3 d$ orbitals and the unoccupied bands comprise an increased $4 s$ and $4 p$ character [89]. A reduction in electronic repulsion stabilizing the core-excited state of laser-heated nickel thus presents a plausible explanation to the spectral red shift observed in the XUV TA spectra. As the electron repulsion and reduction of population in the nickel $3 d$ bands are not linearly proportional to electron temperature, the nonlinearity in the relation between $\omega_{s}$ and $\Delta T$ is also potentially clarified.

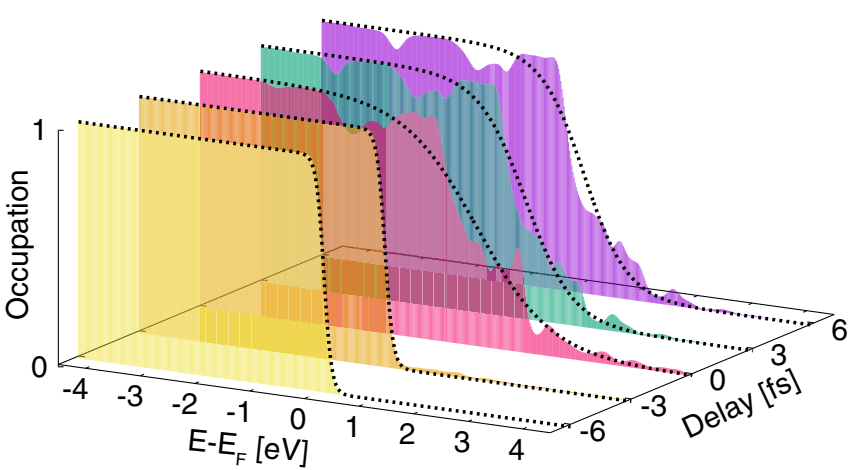

FIG. 6. Simulated electronic occupation as a function of energy during photoexcitation. The results of fitting each time slice to a Fermi-Dirac function are shown in black dashed lines.

\section{B. Nonequilibrium electron relaxation}

While the fluence dependent rise time of the TA signal [Fig. 5(c) and Table I] indicate that the electron thermalization time is longer than the pump pulse duration, no energetically shifting spectral features are observed within the electron thermalization timescale in the TA spectra [Fig. 2(c)] to represent the thermalization of nonequilibrium carrier distribution. To understand this phenomenon, we simulated the dynamics of photoexcitation in nickel through a density matrix formalism based on the band structure of nickel calculated by density functional theory (DFT) (Appendix F). Snapshots of electronic occupation near the Fermi level $\left(E_{F}\right)$ at different time delays with respect to the optical pulse are shown in Fig. 6. In the simulated electron distributions, occupation around $1.5 \mathrm{eV}$ below the Fermi level decreases following photoexcitation. However, the photoexcited electron distribution at and above the Fermi energy still closely resembles a Fermi-Dirac function (Fig. 6, black dashed lines). This implies that because the initial photoexcited electronic occupation does not significantly deviate from a hot thermalized distribution, possible spectral signatures of a nonequilibrium $\mathrm{CB}$ electron distribution will not be easily distinguished. Note that in the density matrix formalism, the effects of scattering between the photoexcited electrons are not included. Therefore it is expected that the true photoexcited carrier distribution will feature an even smaller deviation from the thermalized electron distribution than the simulated results (Fig. 6). This provides a potential explanation to the absence of spectral features of nonthermalized carriers.

\section{CONCLUSION}

In summary, it is observed that the transient absorption spectra of optically excited nickel at the nickel $M_{2,3}$ edge can be simulated with a spectral red shift and Gaussian broadening of the static spectrum. For a hot thermalized electron distribution, the Gaussian broadening is experimentally revealed and theoretically derived to be linearly related to the change of electron temperature and can be used to track the electron temperature. The increase of spectral red shift with rising electron temperature can be plausibly explained by the reduction of electron repulsion due to the repartitioning of localized $3 d$ electrons to $4 s$ and $4 p$ bands through laser 
heating. For thermalized electrons, the red shift displays a power-law relationship with the electron temperature change by a power $\alpha \approx 1.5$. While probing the sub-40-fs dynamics of optically excited nickel, the relation between the spectral shift and electron temperature, and thus the broadening for thermalized carriers, is utilized to determine that the electrons do not thermalize instantaneously during the optical excitation, even though the core-level absorption line shape at sub-15-fs timescale closely resembles the spectra with thermalized electrons. In the core-level transient absorption spectroscopy of nickel at $\mathrm{Ni} M_{2,3}$ edge, the electron thermalization process is represented by a lengthened growth of spectral features for a thermalized electron distribution, indicating that electron scattering and thermalization accompany and follow the photoexcitation and finally create a hot thermalized carrier distribution. A fluence-dependent electron thermalization timescale ranging between $34 \mathrm{fs}$ and $13 \mathrm{fs}$ is extracted by deconvolving the rise of the transient absorption signal from the instrument response.

The results in this work indicate that core-level absorption spectroscopy can be utilized to extract the electron temperature of metallic samples and to assess both the timescale of electron thermalization and the validity of a multitemperature model. As such, this work unifies the observation of $>30$ fs dynamics of thermalized electrons and the fewfemtosecond dynamics of nonequilibrium electron relaxation. The former can be readily probed with time-resolved photoemission methods but has been difficult to extract and interpret from core-level absorption spectroscopy, while the latter can be interrogated with few-femtosecond core-level spectroscopy but is inaccessible through photoemission techniques. The methodology developed within this study facilitates the understanding of core-level absorption spectra of laser-heated metals with a simple and intuitive picture, and the approach can be readily extended to treat other metallic systems or to investigate photoinduced phase transitions in metallic films and multilayers.

\section{ACKNOWLEDGMENTS}

The authors would like to thank Xun Shi, Phoebe Tengdin, Wenjing You, and David Prendergast for fruitful discussions. The experimental laboratory work of S.R.L., H.-T. C., and A.G. was supported by the Defense Advanced Research Projects Agency PULSE Program Grant No. W31P4Q-131-0017 (concluded), the US Air Force Office of Scientific Research Grants No. FA9550-19-1-0314, No. FA9550-20-10334, No. FA9550-15-0037 (concluded), and No. FA955014-1-0154 (concluded), the Army Research Office Grant No. WN911NF-14-1-0383, and The W.M. Keck Foundation Award No. 046300-002. H.-T.C. acknowledges support from Air Force Office of Scientific Research (AFOSR) (Grants No. FA9550-15-1-0037 and No. FA9550-19-1-0314) and W. M. Keck Foundation (Grant No. 046300); A.G. acknowledges support from German Research Foundation (Grant No. GU 1642/1-1) and Air Force Office of Scientific Research (AFOSR) (Grants No. FA9550-15-1-0037 and No. FA955019-1-0314); S.K.C. acknowledges support by the Department of Energy, Office of Energy Efficiency and Renewable Energy (EERE) Postdoctoral Research Award under the EERE
Solar Energy Technologies Office; I.J.P. and S.K.C. acknowledge support from US Department of Energy, Office of Science, Office of Basic Energy Sciences, Materials Sciences and Engineering Division, under Contract No. DEAC0205-CH11231, within the Physical Chemistry of Inorganic Nanostructures Program (KC3103). N.U.D., S.R.A., V.T. and T.S.R. acknowledge support from U.S. Department of Energy (Grant No. DE-FG02-07ER46354). D.M.N. acknowledges support from the Army Research Office under Grant No. W911NF-20-1-0127 and from US Air Force Office of Scientific Research Grant No. FA9550-15-0037 (concluded).

\section{APPENDIX A: EXPERIMENTAL APPARATUS}

The table-top XUV TA setup consists of a Ti:sapphire laser with $1.8 \mathrm{~mJ}$ output pulse energy, $30 \mathrm{fs}$ pulse duration operating at $1 \mathrm{kHz}$ repetition rate. The laser pulses centered at $790 \mathrm{~nm}$ wavelength are then focused into a $1 \mathrm{~m}$ long, Ne filled hollowcore fiber for supercontinuum generation, resulting in pulses with a spectrum spanning between $500 \mathrm{~nm}$ and $1000 \mathrm{~nm}$. A mechanical chopper is installed at the exit of the hollow-core fiber to chop the beam repetition rate down to $100 \mathrm{~Hz}$ so as to prevent sample damage from optical heating due to the poor heat conductivity in nanometer thick thin films. In addition, the sample is raster scanned during the measurement to prevent laser damage due to long time exposure and systematic error due to sample inhomogeneity. Static absorption measurements are taken before and after each transient absorption experiment to assess whether sample damage occurs during the experiment. The spectrally broadened pulses are subsequently compressed by a set of broadband doubleangle chriped mirrors and split by a 1:9 broadband beam splitter into the pump and probe arm, respectively. The third-order dispersion of the laser pulses is compensated by transmitting the beam through a $2 \mathrm{~mm}$ thick ammonium dihydrogen phosphate crystal [90]. The fine tuning of dispersion in the pump and probe arm is achieved by a pair of glass wedges installed in each arm, and the pulse duration of the pump and probe pulses measured by dispersion scan [91] are $4.3 \pm 0.2$ fs and $3.6 \pm 0.1 \mathrm{fs}$ long, respectively. The duration of the pump pulse is slightly longer than the probe because of the limited bandwidth of the broadband beamsplitter. The typical spectrum and temporal profile of the pump pulse are shown in Figs. 7(a) and 7(b), respectively. The intensity of the pump beam is controlled by an iris and the beam is time-delayed, focused, and recombined collinearly into the probe arm with an annular mirror [Fig. 1(a)]. For each individual experiment, the beam profile of the pump is measured by a CMOS camera directly at the sample position for the determination of the intensity and fluence of the pump pulse. The beam in the probe arm is focused into an Ar gas jet to generate broadband XUV pulses spectrally spanning 40-73 eV [Fig. 1(b)]. After filtering the driving near-IR field with a $100 \mathrm{~nm}$ thick $\mathrm{Al}$ filter, the XUV beam is refocused by a gold coated toroidal mirror onto the measurement target. After passing through the measurement target, the XUV beam transmits through another $100 \mathrm{~nm}$ thick Al filter that blocks the pump light and the transmitted XUV is then dispersed by a flat-field grating onto an XUV CCD camera with 16-bit bit depth that provides a dynamic range of approximately 5 orders of magnitude. With 

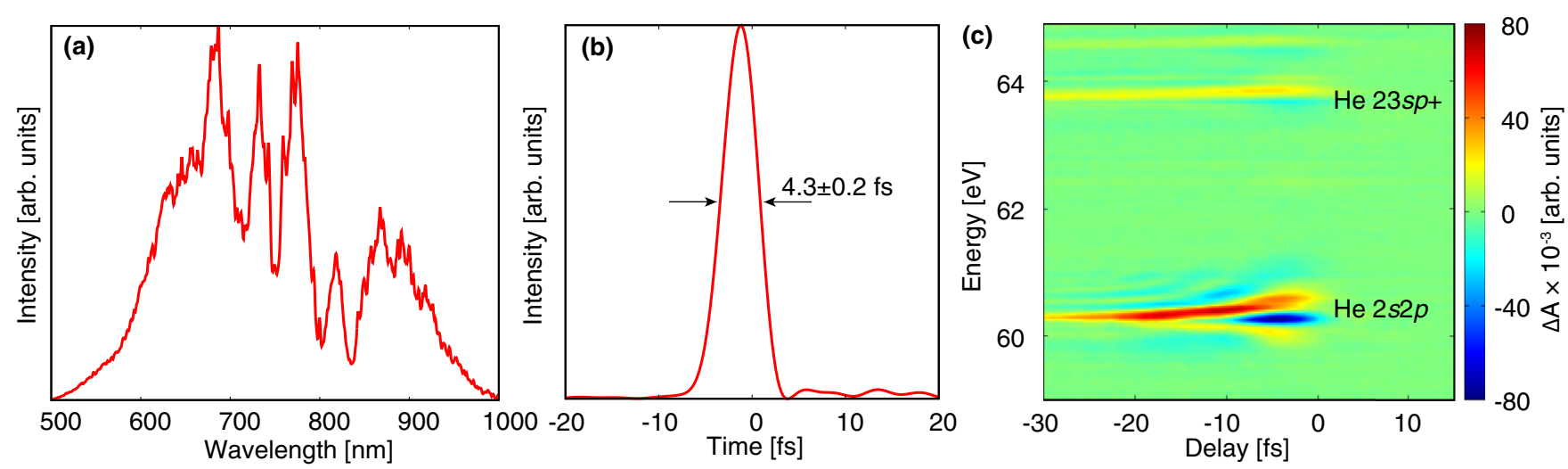

FIG. 7. (a) The typical spectrum and (b) temporal profile of the pump pulses measured by dispersion scan [91]. (c) displays the typical transient absorption spectra of helium $2 s n p$ autoionization states taken subsequently after each scan through all time delay points on the nickel sample for time delay calibration.

the approximately $1 \mathrm{OD}$ (optical density) edge jump at the nickel edge, the noise floor of the transient measurement is approximately $5 \mathrm{mOD}$, which is mainly contributed by the fluctuations of the XUV light source. The spectral resolution of the apparatus at the photon energies of the experiment is approximately $60 \mathrm{meV}$. The measurement delay step sizes used in the results shown in Fig. 1(c) are $1.3 \mathrm{fs}(0.2 \mu \mathrm{m})$ between $-66.7 \mathrm{fs}(-10 \mu \mathrm{m})$ to $+133.3 \mathrm{fs}(+20 \mu \mathrm{m})$ delay, $3.3 \mathrm{fs}$ $(0.5 \mu \mathrm{m})$ between $+133.3 \mathrm{fs}(+20 \mu \mathrm{m})$ and +560 fs $(+84$ $\mu \mathrm{m})$ delay, and $20 \mathrm{fs}(3 \mu \mathrm{m})$ between $+560 \mathrm{fs}(+84 \mu \mathrm{m})$ and $+1.98 \mathrm{ps}(+297 \mu \mathrm{m})$ delay. The measurement delay step size used in the results shown in Fig. 1(d) is $0.33 \mathrm{fs}(0.05 \mu \mathrm{m})$ between $-50 \mathrm{fs}(-7.5 \mu \mathrm{m})$ and $+50 \mathrm{fs}(+7.5 \mu \mathrm{m})$ delay. Here the positions of the optical delay stage in micron are listed in parentheses. As the retroreflector folds the optical beam once [Fig. 1(a)], $1 \mu \mathrm{m}$ change of the optical delay stage translates to approximately 6.6 fs delay.

To avoid the slow drift of time delays during the experiment, we ran one transient absorption measurement on the $2 s n p$ autoionizing states of helium after each scan through all

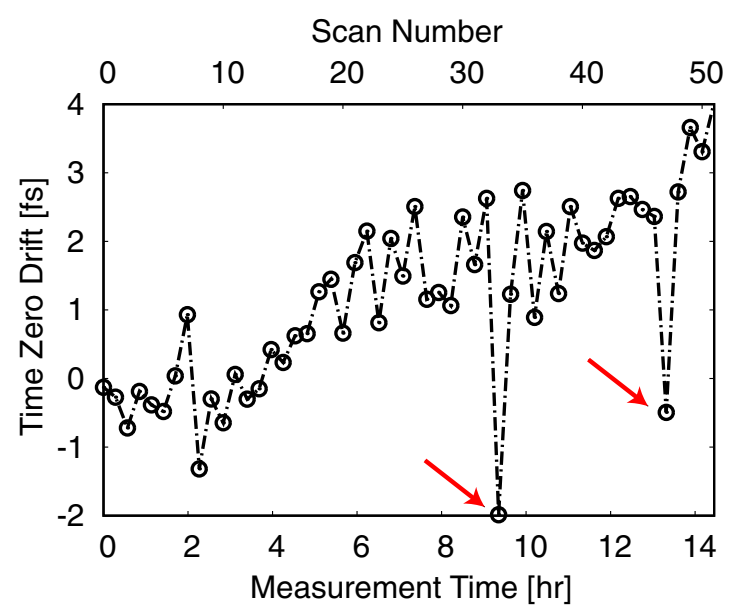

FIG. 8. A sample time zero drift trace taken in an XUV TA experiment on nickel. The time zero is extracted from XUV TA measurements on He autoionization lines. The red arrows indicate the scans to be discarded from data analysis due to $>3$ fs drifts over a single scan. time delay points on the nickel sample [92]. A typical transient absorption trace on the He $2 s n p$ autoionizing states is shown in Fig. 7(c). The transient absorption signal of the He $2 s 2 p$ state near time zero was then fitted to an error function to determine the exact zero time overlap between the XUV and optical pulses. With the calibrated time zero of each scan, the changes of absorbance for each scan were then interpolated onto a grid line and averaged together [40]. A sample time zero drift trace over the course of an XUV TA measurement on nickel is shown in Fig. 8. Note that as the fluence dependence on the TA signal for the He autoionization lines is highly nonlinear [92], reliable TA measurements for time zero correction at fluences lower than $30 \mathrm{~mJ} / \mathrm{cm}^{2}$ have not been obtained. This leads to smearing and stretching of dynamics with respect to time delay for low fluence measurements. To estimate the amount of time-axis smearing in the observed dynamics (e.g., the rise of TA signal shown in Sec. II D) by the time zero drift, XUV TA measurements of He autoionization lines were conducted over the same amount of time as the acquisition time for the XUV TA experiments on nickel and we focus on the overall time delay drift over the entire measurement time. Specifically for the measurements displayed in Table I, an overall time zero drift of $5 \mathrm{fs}$ is obtained. This leads to an increase of negative uncertainty in the first two entries of $\tau_{\text {rise }}$ in Table I.

\section{APPENDIX B: SAMPLE PREPARATION}

The nickel thin films used in this experiment were prepared by dual ion-beam deposition of $50 \mathrm{~nm}$ thick nickel onto $30 \mathrm{~nm}$ thick silicon nitride membranes with a free-standing window size of $3 \mathrm{~mm} \times 3 \mathrm{~mm}$ [93], utilizing (neutralized) $600 \mathrm{eV}$ krypton ions at a background pressure of $10^{-7} \mathrm{~Pa}$. The layer thickness was controlled via the deposition time where typical sputter rates are below $0.1 \mathrm{~nm}$ per second and were calibrated using surface profilometry as well as in situ spectral ellipsometry. The sputter time calculations are based on a numerical model [93], to compensate both interdiffusion losses and systematic deposition variations due to, for example, shutter response times. The substrate holder was rotated during deposition with a spinning frequency of $40 \mathrm{rpm}$ and an $R / \theta$ shaper was used for shaping the particle flux laterally for 
a high lateral homogeneity film thickness growth. A film of $4 \mathrm{~nm}$ thick boron carbide was then deposited above the nickel thin film to prevent oxidation through exposure to ambient air.

\section{APPENDIX C: ESTIMATING ELECTRON TEMPERATURE RISE DUE TO OPTICAL ABSORPTION}

The electron temperature rise after optical excitation of nickel at $40 \mathrm{fs}$ time delay is calculated from the spectrum of the optical pulse [Fig. 1(d)], the wavelength-dependent thin film absorbance, and the electronic heat capacity of nickel. As the electron-phonon scattering time in nickel is a few hundred femtoseconds [63], the electronic cooling due to phonons is neglected in the following calculation. The energy absorbed by the nickel film at a specific wavelength $\lambda$ can be described as $\mathcal{E}_{\text {abs }}(\lambda)=\frac{h c}{\lambda} N_{\text {ph }}(\lambda) f_{\text {abs }}(\lambda)$. Here, $N_{\text {ph }}(\lambda)$ is the number of incident photons as a function of wavelength, which can be directly derived from the pump pulse spectrum. $f_{\text {abs }}(\lambda)$ is the fraction of light absorbed in the nickel film and is calculated using the transfer matrix method [94]. The value of wavelength-dependent complex refractive indices of nickel and silicon nitride are taken from Refs. [95] and [96], respectively. Denoting the electron heat capacity by $C_{e}(T)$, the estimated maximum electron temperature $T_{\text {est }}$ is related to the total energy absorbed by the nickel film $\mathcal{E}$ as

$$
\mathcal{E}=\int_{T_{0}=300 K}^{T_{\text {est }}} C_{e}\left(T^{\prime}\right) d T^{\prime},
$$

where the temperature-dependent electron heat capacity of nickel is taken from Ref. [87]. The electron temperature rise $\Delta T_{\text {est }}$ is $T_{\text {est }}-T_{0}$.

\section{APPENDIX D: EXPANSION OF $I^{\prime}(t)$}

The expression of the core-level absorption line shape $I^{\prime}(\omega)$ in Eq. (2) is the Fourier transform of its free-induction decay

$$
I^{\prime}(t)=\left(\frac{\pi T}{i \sinh (\pi T t)}\right)^{1-\zeta}=(-i \pi S(t, T))^{1-\zeta} .
$$

To find $e^{g(t, \Delta T)}$ [cf. Eq. (4)], we first expand $S(t, T)$ with respect to temperature:

$$
\begin{aligned}
S(t, T+\Delta T)= & S(t, T)+\partial_{T} S(t, T) \Delta T \\
& +\frac{1}{2} \partial_{T}^{2} S(t, T)(\Delta T)^{2}+\ldots
\end{aligned}
$$

Letting $z=\pi t$, the derivatives of $S(t, T)$ are expressed

$$
\begin{aligned}
& \partial_{T} S(t, T)=\frac{1}{\sinh (z T)}(1-z T \operatorname{coth}(z T)) \\
& \partial_{T}^{2} S(t, T)=\frac{T}{\sinh (z T)}\left[2\left(z^{2} \operatorname{coth}^{2}(z T)-\frac{z \operatorname{coth}(z T)}{T}\right)-z^{2}\right] .
\end{aligned}
$$

Expressing the $m$ th Taylor expansion term of $S(t, T+\Delta T)$ as $\partial_{T}^{m} S(t, T)(\Delta T)^{m} / m !=s_{m} S(t, T)$, we can rewrite $S(t, T+$ $\Delta T)$ as

$$
S(t, T+\Delta T)=S(t, T) \exp \left(\ln \left(1+\sum_{m=1}^{\infty} s_{m}\right)\right),
$$

and by comparing the terms between the Taylor expansion of $S(t, T+\Delta T)$ and $\left\{s_{m}\right\}$, the second-order expansion of
$S(t, T+\Delta T)$ can be expressed as $[97,98]$

$$
S(t, T+\Delta T) \approx S(t, T) \exp \left(s_{1}+s_{2}-\frac{s_{1}^{2}}{2}\right) .
$$

Here we concentrate on the behavior of $S(t, T)$ when $t$ is near zero because the denominator $\sinh (z t)$ in the integrand $I^{\prime}(t)$ grows exponentially with $t$. This "short time" approximation can be further justified by comparing the temperature induced broadening and the natural linewidth of the core hole. While the temperature induced broadening is typically on the scale of tens to hundreds of meV [cf. Fig. 3(c)], the core hole lifetime broadening is typically larger by one order of magnitude $[68,99]$. This indicates that the "true" free induction decay of the core-level transitions is far faster than the decay of $I^{\prime}(t)$. Under such condition, we can approximate the term $\operatorname{coth}(z T)$ using the asymptotic relation $\operatorname{coth} x \approx 1 / x+x / 3-x^{3} / 45+$ ... [100]. To first order with $\operatorname{coth}(z T) \approx 1 /(z T)$, we obtain

$$
\begin{aligned}
s_{1} & =\left(\frac{1}{T}-z \operatorname{coth}(z T)\right) \Delta T \approx 0 \\
s_{2} & =\left(z^{2} \operatorname{coth}^{2}(z T)-\frac{z \operatorname{coth}(z T)}{T}-\frac{z^{2}}{2}\right)(\Delta T)^{2} \\
& \approx-\frac{z^{2}}{2}(\Delta T)^{2} .
\end{aligned}
$$

Inserting the expansion terms back into Eq. (D2), a preliminary expression for $S(t, T+\Delta T)$ is derived:

$$
S(t, T+\Delta T) \approx S(t, T) \exp \left(-\frac{(\pi \Delta T t)^{2}}{2}\right),
$$

and therefore

$$
e^{g(t, \Delta T)}=\exp \left(-\frac{(\pi \Delta T t)^{2}}{2}\right) .
$$

To estimate the error of the approximated expression, we include the second and the third term of the asymptotic expansion $\left(\operatorname{coth}(z T) \approx 1 /(z T)+z T / 3-(z T)^{3} / 45\right)$ and obtain

$$
\begin{aligned}
s_{1} & =\left(\frac{1}{T}-z \operatorname{coth}(z T)\right) \Delta T \approx\left(-\frac{z^{2} T}{3}+\frac{z^{4} T^{3}}{45}\right) \Delta T, \\
s_{2} & =\left(z^{2} \operatorname{coth}^{2}(z T)-\frac{z \operatorname{coth}(z T)}{T}-\frac{z^{2}}{2}\right)(\Delta T)^{2} \\
& \approx\left(-\frac{z^{2}}{2}+\frac{z^{2}}{3}+\frac{4 z^{4} T^{2}}{45}\right)(\Delta T)^{2} .
\end{aligned}
$$

The expression $S(t, T+\Delta T)$ becomes

$$
\begin{aligned}
S(t, T+\Delta T) \approx & S(t, T) \exp \left(-z^{2}\left(\frac{\Delta T^{2}+2 T \Delta T}{6}\right)\right) \\
& \times \exp \left(z^{4}\left(\frac{T^{3} \Delta T}{45}+\frac{T^{2}(\Delta T)^{2}}{30}\right)\right) .
\end{aligned}
$$

Note that in the equations above, we only include the terms up to $z^{4}$ because in the region where $z=\pi t$ is near zero, the higher-order terms can be neglected. The same reasoning also applies to leaving out the higher-order terms in the asymptotic expansion of $\operatorname{coth}(z T)$ as higher-order terms will lead to expressions of $z^{6}$ and above in $S(t, T+\Delta T)$. To explore the adequacy of the "short time" approximation, we compare 
the magnitude of the $z^{2}$ term to the $z^{4}$ term. Given the typical core hole lifetime broadening of $\sim 1 \mathrm{eV}$ [99], room temperature $T \approx 0.03 \mathrm{eV}$, and an overestimated $\Delta T=10000 \mathrm{~K}$ $\approx 0.9 \mathrm{eV}$, the magnitude of the second-order term is

$$
\left(\frac{\pi \hbar}{1 \mathrm{eV}}\right)^{2} \frac{0.9^{2}+2 \times 0.9 \times 0.03}{6 \hbar^{2}}(\mathrm{eV})^{2} \approx 1.42
$$

and the magnitude of the $z^{4}$ term is

$$
\left(\frac{\pi \hbar}{1 \mathrm{eV}}\right)^{4}\left(\frac{0.9 \times 0.03^{3}}{45}+\frac{0.03^{2} \times 0.9^{2}}{30}\right)\left(\frac{\mathrm{eV}}{\hbar}\right)^{4} \approx 0.002
$$

The magnitude of the fourth-order term is three orders of magnitude smaller than the second-order term, indicating that the $z^{4}$ term can also be neglected, yielding

$$
S(t, T+\Delta T) \approx S(t, T) \exp \left(-\frac{\left(\Delta T^{2}+2 T \Delta T\right)(\pi t)^{2}}{6}\right) .
$$

Here we observe that the expression remains a Gaussian function with respect to $t$ and there is no first-order term with respect to $t$ in the exponent. The Fourier transformed broadening factor in the spectral domain is proportional to $\sqrt{\Delta T^{2}+2 T \Delta T}$. However, as the electron temperature change $\Delta T$ is at least 6 times larger than the temperature for the reference spectrum $T=T_{0}=300 \mathrm{~K}$ in the measurements [Fig. 3(c)], the term $2 T \Delta T$ is small compared to $\Delta T^{2}$. This explains the linear relationship between the obtained spectral broadening and electron temperature. As a corollary on the comparison of terms with different orders of $z$, note that the terms in the expansion of $\left\{s_{m}\right\}$ always have the form $z^{n} T^{k}(\Delta T)^{l}$, with $n, k, l \geqslant 0$ and $k+l=n$. This indicates that the "short time" approximation, or the truncation at the second order holds as long as the electron temperature change $\left(k_{B} \Delta T\right)$ and electron temperature $\left(k_{B} T\right)$ are smaller than the core hole broadening.

Finally, we connect Eq. (D3) with Eq. (4) and observe

$$
\begin{aligned}
\frac{\pi\left(T_{0}+\Delta T\right)}{\sinh \left(\pi\left(T_{0}+\Delta T\right) t\right)} & \approx S\left(t, T_{0}\right) \times \exp \left(-\frac{\Delta T^{2}}{6}(\pi t)^{2}\right) \\
& \approx \frac{\pi T_{0}}{\sinh \left(\pi T_{0} t\right)} \times e^{g(t, \Delta T)},
\end{aligned}
$$

indicating $g(t, \Delta T) \approx \frac{(\pi \Delta T)^{2}}{6}$. In the equation above, the term $2 T \Delta T$ from Eq. (D3) is ignored as $(\Delta T)^{2} \gg 2 T \Delta T$. The broadening function $f(\omega, \Delta T)=\frac{1}{2 \pi} \int_{-\infty}^{\infty} e^{i \omega t} e^{(1-\zeta) g(t, \Delta T)} d t$ is thus

$$
f(\omega, \Delta T) \approx \frac{1}{\sigma \sqrt{2 \pi}} e^{-\frac{\omega^{2}}{2 \sigma^{2}}}
$$

with

$$
\sigma=\pi \Delta T \sqrt{\frac{1-\zeta}{3}} .
$$

Rewriting the equation in SI units, the broadening function reads

$$
f(\hbar \omega, \Delta T) \approx \frac{1}{\sigma \sqrt{2 \pi}} e^{-\frac{(\hbar \omega)^{2}}{2 \sigma^{2}}}
$$

and

$$
\sigma=\pi k_{B} \Delta T \sqrt{\frac{1-\zeta}{3}}=a \Delta T .
$$

Here $a=\pi k_{B} \sqrt{(1-\zeta) / 3}$ and $\zeta<1$ is a constant phase factor related to the electron scattering from the core hole potential [Eq. (2)].

\section{APPENDIX E: FITTING WITH MODIFIED GAUSSIAN ERROR FUNCTION}

To fit the sub-20-fs dynamics of $\sigma(t), \omega_{s}(t)$, and the TA lineouts at 65.75 and $67.4 \mathrm{eV}$, a modified Gaussian error function

$$
\mathcal{F}(t, \tau)=\frac{c_{1}}{2}\left(1+\frac{2}{\sqrt{\pi}} \int_{t_{0}}^{t} e^{-\left(\frac{2 \sqrt{\ln 2\left(t^{\prime}-t_{0}\right)}}{\tilde{\tau}}\right)^{2}} d t^{\prime}\right)+c_{0}
$$

is utilized, where $t_{0}$ marks the timing of the dynamics relative to zero time delay and $\tilde{\tau}$ the duration of the growth; $c_{0}$ and $c_{1}$ are fitting coefficients for offset and amplitude of the TA signal. The coefficient $2 \sqrt{\ln 2}$ enables direct comparison between $\tilde{\tau}$ and the cross-correlation time between the pump and probe pulses. If the electronic response is instantaneous with respect to the excitation pulse, $\tilde{\tau}$ will be equal to the cross-correlation time.

\section{APPENDIX F: SIMULATION OF PHOTOEXCITATION DYNAMICS}

The dynamics of photoexcitation are simulated by the density matrix formalism based on the nickel band structure calculated by DFT, where the density matrix $\rho_{\mathbf{x x}^{\prime}}(t)=$ $\left\langle c_{\mathbf{x}}^{\dagger}(t) c_{\mathbf{x}^{\prime}}(t)\right\rangle . c$ is the annihilation operator and $\mathbf{x}=\{\mathbf{k}, m, s\}$ denotes the combination of momentum $\mathbf{k}$, band index $m$, and spin index $s$. The Hamiltonian for the Liouville equation $\dot{\rho}=-i[H, \rho] / \hbar$ is

$$
H=\sum_{\mathbf{x}} \mathcal{E}_{\mathbf{x}} c_{\mathbf{x}}^{\dagger} c_{\mathbf{x}}+\sum_{\mathbf{x}, \mathbf{x}^{\prime}} V_{\mathbf{x}, \mathbf{x}^{\prime}}(t) c_{\mathbf{x}}^{\dagger} c_{\mathbf{x}^{\prime}},
$$

where $\mathcal{E}$ denotes the band energy and

$$
V_{\mathbf{x}, \mathbf{x}^{\prime}}(t)=-\mathbf{d}_{\mathbf{x}, \mathbf{x}^{\prime}} \cdot \mathbf{E}(t) .
$$

In the equation above, $\mathbf{d}$ is the dipole operator and the magnitude of the electric field $\mathbf{E}(t)$ is $E_{0} \exp \left(-\left(2 \sqrt{\ln 2} t / \tau_{\text {pulse }}\right)^{2}\right)$. $E_{0}=2.5 \mathrm{~V} / \mathrm{nm}$ is derived from the peak intensity of the pulse and $\tau_{\text {pulse }}=4.3$ fs. To obtain band energies $\mathcal{E}_{\mathbf{x}}$ and dipole operator $\mathbf{d}_{\mathbf{x}, \mathbf{x}^{\prime}}$, DFT calculations were performed using the QUANTUM ESPRESSO package with Perdew-Burke-Ernzerhof (PBE) exchange correlation functional and ultrasoft, scalar relativistic pseudopotential [101-103]. The simulation was conducted on a $15 \times 15 \times 15 k$-point meshgrid using the Monkhorst-Pack scheme [104], and converged with cutoff energy at 60 Ryd. The occupation number as a function of energy and time delay $O(\mathcal{E}, t)$ is calculated by summing the mapping of the diagonal terms of the density matrix onto an energy grid and subsequently dividing by the density of states:

$$
O(\mathcal{E}, t)=\sum_{\mathbf{x}} w_{\mathbf{x}} \rho_{\mathbf{x x}}(t) M\left(\mathcal{E}, \mathcal{E}_{\mathbf{x}}\right) / \sum_{\mathbf{x}} w_{\mathbf{x}} M\left(\mathcal{E}, \mathcal{E}_{\mathbf{x}}\right) .
$$


We use a Gaussian mapping function $M\left(\mathcal{E}, \mathcal{E}_{\mathbf{x}}\right)=$ $\exp \left(-\left(\left(\mathcal{E}-\mathcal{E}_{\mathbf{x}}\right) / \delta \mathcal{E}\right)^{2}\right)$ with width $\delta \mathcal{E}=0.1 \mathrm{eV} . \quad w_{\mathbf{x}}$ is the weighting coefficient within the Monkhorst-Pack scheme at point $\mathbf{k}$.
[1] R. T. Ross and A. J. Nozik, J. Appl. Phys. 53, 3813 (1982).

[2] P. Würfel, Sol. Energy Mater. Sol. Cells 46, 43 (1997).

[3] A. Luque and A. Martí, Sol. Energy Mater. Sol. Cells 94, 287 (2010).

[4] A. E. Jailaubekov, A. P. Willard, J. R. Tritsch, W.-L. Chan, N. Sai, R. Gearba, L. G. Kaake, K. J. Williams, K. Leung, P. J. Rossky, and X.-Y. Zhu, Nat. Mater. 12, 66 (2013).

[5] K. Kamide, T. Mochizuki, H. Akiyama, and H. Takato, Phys. Rev. Appl. 10, 044069 (2018).

[6] Y. Zhang, S. He, W. Guo, Y. Hu, J. Huang, J. R. Mulcahy, and W. D. Wei, Chem. Rev. 118, 2927 (2018).

[7] Z. Zhang, C. Zhang, H. Zheng, and H. Xu, Acc. Chem. Res. 52, 2506 (2019).

[8] E. Beaurepaire, J.-C. Merle, A. Daunois, and J.-Y. Bigot, Phys. Rev. Lett. 76, 4250 (1996).

[9] M. Imada, A. Fujimori, and Y. Tokura, Rev. Mod. Phys. 70, 1039 (1998).

[10] A. Kirilyuk, A. V. Kimel, and T. Rasing, Rev. Mod. Phys. 82, 2731 (2010).

[11] S. L. Johnson, M. Savoini, P. Beaud, G. Ingold, U. Staub, F. Carbone, L. Castiglioni, M. Hengsberger, and J. Osterwalder, Struct. Dyn. 4, 061506 (2017).

[12] J. Shah, Ultrafast Spectroscopy of Semiconductors and Semiconductornanostructures, Springer Series in Solid-State Sciences No. 115 (Springer, Berlin, New York, 1996).

[13] S. I. Anisimov, B. L. Kapeliovich, and T. L. Perelman, Sov. JETP 39, 375 (1974).

[14] P. B. Allen, Phys. Rev. Lett. 59, 1460 (1987).

[15] N. Singh, Int. J. Mod. Phys. B 24, 1141 (2010).

[16] T. Vasileiadis, L. Waldecker, D. Foster, A. Da Silva, D. Zahn, R. Bertoni, R. E. Palmer, and R. Ernstorfer, ACS Nano 12, 7710 (2018).

[17] T. Kampfrath, L. Perfetti, F. Schapper, C. Frischkorn, and M. Wolf, Phys. Rev. Lett. 95, 187403 (2005).

[18] B. Mansart, M. J. G. Cottet, G. F. Mancini, T. Jarlborg, S. B. Dugdale, S. L. Johnson, S. O. Mariager, C. J. Milne, P. Beaud, S. Grübel, J. A. Johnson, T. Kubacka, G. Ingold, K. Prsa, H. M. Rønnow, K. Conder, E. Pomjakushina, M. Chergui, and F. Carbone, Phys. Rev. B 88, 054507 (2013).

[19] H.-S. Rhie, H. A. Dürr, and W. Eberhardt, Phys. Rev. Lett. 90, 247201 (2003).

[20] A. V. Kimel, A. Kirilyuk, A. Tsvetkov, R. V. Pisarev, and T. Rasing, Nature (London) 429, 850 (2004).

[21] C. Stamm, T. Kachel, N. Pontius, R. Mitzner, T. Quast, K. Holldack, S. Khan, C. Lupulescu, E. F. Aziz, M. Wietstruk, H. A. Dürr, and W. Eberhardt, Nat. Mater. 6, 740 (2007).

[22] T. Kachel, N. Pontius, C. Stamm, M. Wietstruk, E. F. Aziz, H. A. Dürr, W. Eberhardt, and F. M. F. de Groot, Phys. Rev. B 80, 092404 (2009).

[23] C. Boeglin, E. Beaurepaire, V. Halté, V. López-Flores, C. Stamm, N. Pontius, H. A. Dürr, and J.-Y. Bigot, Nature (London) 465, 458 (2010).

[24] C. La-O-Vorakiat, E. Turgut, C. A. Teale, H. C. Kapteyn, M. M. Murnane, S. Mathias, M. Aeschlimann, C. M.
Schneider, J. M. Shaw, H. T. Nembach, and T. J. Silva, Phys. Rev. X 2, 011005 (2012).

[25] I. Radu, K. Vahaplar, C. Stamm, T. Kachel, N. Pontius, H. A. Dürr, T. A. Ostler, J. Barker, R. F. L. Evans, R. W. Chantrell, A. Tsukamoto, A. Itoh, A. Kirilyuk, T. Rasing, and A. V. Kimel, Nature (London) 472, 205 (2011).

[26] U. Staub, R. A. de Souza, P. Beaud, E. Möhr-Vorobeva, G. Ingold, A. Caviezel, V. Scagnoli, B. Delley, W. F. Schlotter, J. J. Turner, O. Krupin, W.-S. Lee, Y.-D. Chuang, L. Patthey, R. G. Moore, D. Lu, M. Yi, P. S. Kirchmann, M. Trigo, P. Denes, D. Doering, Z. Hussain, Z. X. Shen, D. Prabhakaran, A. T. Boothroyd, and S. L. Johnson, Phys. Rev. B 89, 220401(R) (2014).

[27] J. A. Johnson, T. Kubacka, M. C. Hoffmann, C. Vicario, S. de Jong, P. Beaud, S. Grübel, S.-W. Huang, L. Huber, Y. W. Windsor, E. M. Bothschafter, L. Rettig, M. Ramakrishnan, A. Alberca, L. Patthey, Y.-D. Chuang, J. J. Turner, G. L. Dakovski, W.-S. Lee, M. P. Minitti, W. Schlotter, R. G. Moore, C. P. Hauri, S. M. Koohpayeh, V. Scagnoli, G. Ingold, S. L. Johnson, and U. Staub, Phys. Rev. B 92, 184429 (2015).

[28] M. Naseska, A. Pogrebna, G. Cao, Z. A. Xu, D. Mihailovic, and T. Mertelj, Phys. Rev. B 98, 035148 (2018).

[29] A. V. Kimel, A. Kirilyuk, P. A. Usachev, R. V. Pisarev, A. M. Balbashov, and T. Rasing, Nature (London) 435, 655 (2005).

[30] J.-Y. Bigot, M. Vomir, and E. Beaurepaire, Nat. Phys. 5, 515 (2009).

[31] C. E. Graves, A. H. Reid, T. Wang, B. Wu, S. de Jong, K. Vahaplar, I. Radu, D. P. Bernstein, M. Messerschmidt, L. Müller, R. Coffee, M. Bionta, S. W. Epp, R. Hartmann, N. Kimmel, G. Hauser, A. Hartmann, P. Holl, H. Gorke, J. H. Mentink, A. Tsukamoto, A. Fognini, J. J. Turner, W. F. Schlotter, D. Rolles, H. Soltau, L. Strüder, Y. Acremann, A. V. Kimel, A. Kirilyuk, T. Rasing, J. Stöhr, A. O. Scherz, and H. A. Dürr, Nat. Mater. 12, 293 (2013).

[32] A. Eschenlohr, M. Battiato, P. Maldonado, N. Pontius, T. Kachel, K. Holldack, R. Mitzner, A. Föhlisch, P. M. Oppeneer, and C. Stamm, Nat. Mater. 12, 332 (2013).

[33] G. Batignani, D. Bossini, N. Di Palo, C. Ferrante, E. Pontecorvo, G. Cerullo, A. Kimel, and T. Scopigno, Nat. Photonics 9, 506 (2015).

[34] D. Bossini, S. Dal Conte, Y. Hashimoto, A. Secchi, R. V. Pisarev, T. Rasing, G. Cerullo, and A. V. Kimel, Nat. Commun. 7, 10645 (2016).

[35] F. Siegrist, J. A. Gessner, M. Ossiander, C. Denker, Y.-P. Chang, M. C. Schröder, A. Guggenmos, Y. Cui, J. Walowski, U. Martens, J. K. Dewhurst, U. Kleineberg, M. Münzenberg, S. Sharma, and M. Schultze, Nature (London) 571, 240 (2019).

[36] M. Obergfell and J. Demsar, Phys. Rev. Lett. 124, 037401 (2020).

[37] A. El-Ghazaly, B. Tran, A. Ceballos, C.-H. Lambert, A. Pattabi, S. Salahuddin, F. Hellman, and J. Bokor, Appl. Phys. Lett. 114, 232407 (2019).

[38] M. Hofherr, S. Häuser, J. K. Dewhurst, P. Tengdin, S. Sakshath, H. T. Nembach, S. T. Weber, J. M. Shaw, T. J. Silva, 
H. C. Kapteyn, M. Cinchetti, B. Rethfeld, M. M. Murnane, D. Steil, B. Stadtmüller, S. Sharma, M. Aeschlimann, and S. Mathias, Sci. Adv. 6, eaay8717 (2020).

[39] M. Schultze, K. Ramasesha, C. D. Pemmaraju, S. A. Sato, D. Whitmore, A. Gandman, J. S. Prell, L. J. Borja, D. Prendergast, K. Yabana, D. M. Neumark, and S. R. Leone, Science 346, 1348 (2014).

[40] M. Zürch, H.-T. Chang, L. J. Borja, P. M. Kraus, S. K. Cushing, A. Gandman, C. J. Kaplan, M. H. Oh, J. S. Prell, D. Prendergast, C. D. Pemmaraju, D. M. Neumark, and S. R. Leone, Nat. Commun. 8, 15734 (2017).

[41] M. Zürch, H.-T. Chang, P. M. Kraus, S. K. Cushing, L. J. Borja, A. Gandman, C. J. Kaplan, M. H. Oh, J. S. Prell, D. Prendergast, C. D. Pemmaraju, D. M. Neumark, and S. R. Leone, Struct. Dyn. 4, 044029 (2017).

[42] F. Schlaepfer, M. Lucchini, S. A. Sato, M. Volkov, L. Kasmi, N. Hartmann, A. Rubio, L. Gallmann, and U. Keller, Nat. Phys. 14, 560 (2018).

[43] M. F. Lin, M. A. Verkamp, J. Leveillee, E. S. Ryland, K. Benke, K. Zhang, C. Weninger, X. Shen, R. Li, D. Fritz, U. Bergmann, X. Wang, A. Schleife, and J. Vura-Weis, J. Phys. Chem. C 121, 27886 (2017).

[44] M. A. Verkamp, J. Leveillee, A. Sharma, A. Schleife, and J. Vura-Weis, ChemRxiv (2019), doi: 10.26434/chemrxiv.8323289.v1.

[45] L. M. Carneiro, S. K. Cushing, C. Liu, Y. Su, P. Yang, A. P. Alivisatos, and S. R. Leone, Nat. Mater. 16, 819 (2017).

[46] S. K. Cushing, M. Zürch, P. M. Kraus, L. M. Carneiro, A. Lee, H.-T. Chang, C. J. Kaplan, and S. R. Leone, Struct. Dyn. 5, 054302 (2018).

[47] I. J. Porter, S. K. Cushing, L. M. Carneiro, A. Lee, J. C. Ondry, J. C. Dahl, H.-T. Chang, A. P. Alivisatos, and S. R. Leone, J. Phys. Chem. Lett. 9, 4120 (2018).

[48] S. K. Cushing, A. Lee, I. J. Porter, L. M. Carneiro, H.-T. Chang, M. Zürch, and S. R. Leone, J. Phys. Chem. C 123, 3343 (2019)

[49] S. K. Cushing, I. J. Porter, B. R. de Roulet, A. Lee, B. M. Marsh, S. Szoke, M. E. Vaida, and S. R. Leone, Sci. Adv. 6, eaay6650 (2020).

[50] M. Volkov, S. A. Sato, F. Schlaepfer, L. Kasmi, N. Hartmann, M. Lucchini, L. Gallmann, A. Rubio, and U. Keller, Nat. Phys. 15, 1145 (2019).

[51] J. J. Rehr, Found. Phys. 33, 1735 (2003).

[52] A. R. Attar, H.-T. Chang, A. Britz, X. Zhang, M.-F. Lin, A. Krishnamoorthy, T. Linker, D. Fritz, D. M. Neumark, R. K. Kalia, A. Nakano, P. Ajayan, P. Vashishta, U. Bergmann, and S. R. Leone, ACS Nano 14, 15829 (2020).

[53] G. D. Mahan, Many-Particle Physics (Springer US, Boston, MA, 2000).

[54] G. D. Mahan, Phys. Rev. 163, 612 (1967).

[55] B. Roulet, J. Gavoret, and P. Nozières, Phys. Rev. 178, 1072 (1969).

[56] P. Nozières and C. T. De Dominicis, Phys. Rev. 178, 1097 (1969).

[57] G. D. Mahan, Phys. Rev. B 11, 4814 (1975).

[58] K. Ohtaka and Y. Tanabe, Rev. Mod. Phys. 62, 929 (1990).

[59] J. Hohlfeld, E. Matthias, R. Knorren, and K. H. Bennemann, Phys. Rev. Lett. 78, 4861 (1997).

[60] U. Conrad, J. Güdde, V. Jähnke, and E. Matthias, Appl. Phys. B 68, 511 (1999).
[61] H. Regensburger, R. Vollmer, and J. Kirschner, Phys. Rev. B 61, 14716 (2000).

[62] A. Melnikov, J. Güdde, and E. Matthias, Appl. Phys. B 74, 735 (2002).

[63] M. van Kampen, J. T. Kohlhepp, W. J. M. de Jonge, B. Koopmans, and R. Coehoorn, J. Phys. Condens. Matter 17, 6823 (2005).

[64] W. You, P. Tengdin, C. Chen, X. Shi, D. Zusin, Y. Zhang, C. Gentry, A. Blonsky, M. Keller, P. M. Oppeneer, H. Kapteyn, Z. Tao, and M. Murnane, Phys. Rev. Lett. 121, 077204 (2018).

[65] P. Tengdin, W. You, C. Chen, X. Shi, D. Zusin, Y. Zhang, C. Gentry, A. Blonsky, M. Keller, P. M. Oppeneer, H. C. Kapteyn, Z. Tao, and M. M. Murnane, Sci. Adv. 4, eaap9744 (2018).

[66] P. Maldonado, T. Chase, A. H. Reid, X. Shen, R. K. Li, K. Carva, T. Payer, M. Horn von Hoegen, K. Sokolowski-Tinten, X. J. Wang, P. M. Oppeneer, and H. A. Dürr, Phys. Rev. B 101, 100302(R) (2020).

[67] B. Y. Mueller and B. Rethfeld, Phys. Rev. B 87, 035139 (2013).

[68] R. E. Dietz, E. G. McRae, and J. H. Weaver, Phys. Rev. B 21, 2229 (1980).

[69] K. Carva, D. Legut, and P. M. Oppeneer, Europhys. Lett. 86, 57002 (2009).

[70] R. E. Dietz, E. G. McRae, Y. Yafet, and C. W. Caldwell, Phys. Rev. Lett. 33, 1372 (1974).

[71] L. C. Davis and L. A. Feldkamp, Solid State Commun. 19, 413 (1976).

[72] S. Valencia, A. Kleibert, A. Gaupp, J. Rusz, D. Legut, J. Bansmann, W. Gudat, and P. M. Oppeneer, Phys. Rev. Lett. 104, 187401 (2010)

[73] C.-O. Almbladh and P. Minnhagen, Phys. Status Solidi B 85, 135 (1978).

[74] K. Ohtaka and Y. Tanabe, Phys. Rev. B 28, 6833 (1983).

[75] Y. Tanabe and K. Ohtaka, Phys. Rev. B 29, 1653 (1984).

[76] K. Ohtaka and Y. Tanabe, Phys. Rev. B 30, 4235 (1984).

[77] V. M. Adamjan, J. Ortner, A. G. Salistra, and I. M. Tkachenko, Phys. Rev. B 52, 13827 (1995).

[78] J. Ortner, Phys. Rev. B 54, 4401 (1996).

[79] J. Ortner, Phys. B Condens. Matter 239, 328 (1997).

[80] B. Johansson and N. Mårtensson, Phys. Rev. B 21, 4427 (1980).

[81] C. G. Olson and D. W. Lynch, Solid State Commun. 36, 513 (1980).

[82] C. G. Olson and D. W. Lynch, Solid State Commun. 33, 849 (1980).

[83] L. Waldecker, R. Bertoni, R. Ernstorfer, and J. Vorberger, Phys. Rev. X 6, 021003 (2016).

[84] D. Pines and P. Nozières, The Theory of Quantum Liquids, Advanced Book Classics Series (Addison-Wesley, Advanced Book Program, Redwood City, Calif, 1989).

[85] N. Rothenbach, M. E. Gruner, K. Ollefs, C. Schmitz-Antoniak, S. Salamon, P. Zhou, R. Li, M. Mo, S. Park, X. Shen, S. Weathersby, J. Yang, X. J. Wang, R. Pentcheva, H. Wende, U. Bovensiepen, K. Sokolowski-Tinten, and A. Eschenlohr, Phys. Rev. B 100, 174301 (2019).

[86] Z. Lin and L. V. Zhigilei, Appl. Surf. Sci. 253, 6295 (2007).

[87] Z. Lin, L. V. Zhigilei, and V. Celli, Phys. Rev. B 77, 075133 (2008). 
[88] E. Bévillon, J. P. Colombier, V. Recoules, and R. Stoian, Phys. Rev. B 89, 115117 (2014).

[89] Springer Handbook of Electronic and Photonic Materials, edited by S. Kasap and P. Capper, 2nd ed., Springer Handbooks (Springer, Cham, Switzerland, 2017).

[90] H. Timmers, Y. Kobayashi, K. F. Chang, M. Reduzzi, D. M. Neumark, and S. R. Leone, Opt. Lett. 42, 811 (2017).

[91] F. Silva, M. Miranda, B. Alonso, J. Rauschenberger, V. Pervak, and H. Crespo, Opt. Express 22, 10181 (2014).

[92] A. Kaldun, A. Blättermann, V. Stooß, S. Donsa, H. Wei, R. Pazourek, S. Nagele, C. Ott, C. D. Lin, J. Burgdörfer, and T. Pfeifer, Science 354, 738 (2016).

[93] A. Guggenmos, R. Rauhut, M. Hofstetter, S. Hertrich, B. Nickel, J. Schmidt, E. M. Gullikson, M. Seibald, W. Schnick, and U. Kleineberg, Opt. Express 21, 21728 (2013).

[94] G. F. Burkhard, E. T. Hoke, and M. D. McGehee, Adv. Mater. 22, 3293 (2010).

[95] P. Johnson and R. Christy, Phys. Rev. B 9, 5056 (1974).

[96] M. R. Vogt, Development of physical models for the simulation of optical properties of solar cell modules, Ph.D. thesis, Dissertation, Gottfried Wilhelm Leibniz Universität Hannover, 2015.

[97] M. Lax, J. Chem. Phys. 20, 1752 (1952).

[98] R. Kubo, J. Phys. Soc. Jpn. 17, 1100 (1962).

[99] F. de Groot and A. Kotani, Core Level Spectroscopy of Solids, Advances in Condensed Matter Science Vol. 6 (CRC, Boca Raton, 2008).
[100] M. Abramowitz and I. A. Stegun (Eds.), Handbook of Mathematical Functions: With Formulas, Graphs, and Mathematical Tables, Dover Books on Advanced Mathematics (Dover Publications, New York, 1970).

[101] J. P. Perdew, K. Burke, and M. Ernzerhof, Phys. Rev. Lett. 77, 3865 (1996).

[102] P. Giannozzi, S. Baroni, N. Bonini, M. Calandra, R. Car, C. Cavazzoni, D. Ceresoli, G. L. Chiarotti, M. Cococcioni, I. Dabo, A. Dal Corso, S. de Gironcoli, S. Fabris, G. Fratesi, R. Gebauer, U. Gerstmann, C. Gougoussis, A. Kokalj, M. Lazzeri, L. Martin-Samos, N. Marzari, F. Mauri, R. Mazzarello, S. Paolini, A. Pasquarello, L. Paulatto, C. Sbraccia, S. Scandolo, G. Sclauzero, A. P. Seitsonen, A. Smogunov, P. Umari, and R. M. Wentzcovitch, J. Phys. Condens. Matter 21, 395502 (2009).

[103] P. Giannozzi, O. Andreussi, T. Brumme, O. Bunau, M. Buongiorno Nardelli, M. Calandra, R. Car, C. Cavazzoni, D. Ceresoli, M. Cococcioni, N. Colonna, I. Carnimeo, A. Dal Corso, S. de Gironcoli, P. Delugas, R. A. DiStasio, A. Ferretti, A. Floris, G. Fratesi, G. Fugallo, R. Gebauer, U. Gerstmann, F. Giustino, T. Gorni, J. Jia, M. Kawamura, H.-Y. Ko, A. Kokalj, E. Küçükbenli, M. Lazzeri, M. Marsili, N. Marzari, F. Mauri, N. L. Nguyen, H.-V. Nguyen, A. Oterode-la-Roza, L. Paulatto, S. Poncé, D. Rocca, R. Sabatini, B. Santra, M. Schlipf, A. P. Seitsonen, A. Smogunov, I. Timrov, T. Thonhauser, P. Umari, N. Vast, X. Wu, and S. Baroni, J. Phys. Condens. Matter 29, 465901 (2017).

[104] H. J. Monkhorst and J. D. Pack, Phys. Rev. B 13, 5188 (1976). 